\title{
Magnetoelastic coupling behavior at the ferromagnetic transition in the partially disordered double perovskite $\mathrm{La}_{2} \mathrm{NiMnO}_{6}$
}

\author{
Dexin Yang, ${ }^{1,2}$ Giulio I. Lampronti, ${ }^{2}$ C. R. Sebastian Haines, ${ }^{2}$ and Michael A. Carpenter ${ }^{2}$ \\ ${ }^{1}$ College of Materials \& Environmental Engineering, Hangzhou Dianzi University, Hangzhou 310018, People's Republic of China \\ ${ }^{2}$ Department of Earth Sciences, University of Cambridge, Downing Street, Cambridge CB2 3EQ, United Kingdom
}

(Received 19 March 2019; revised manuscript received 21 May 2019; published 23 July 2019)

\begin{abstract}
The magnetocapacitance and magnetoresistance properties near room temperature of partially disordered double perovskite $\mathrm{La}_{2} \mathrm{NiMnO}_{6}$ are related, at least in part, to coupled ferroelastic and magnetic instabilities that are responsible for a ferromagnetic phase transition near $280 \mathrm{~K}$. A systematic analysis of this coupling from the perspectives of strain and elasticity has revealed a system with biquadratic coupling among three order parameters belonging to irreducible representations of $X_{3}^{+}, \Gamma_{4}^{+}$and $\mathrm{m} \Gamma_{4}^{+}$of the parent space group $F m \overline{3} m$. Classical octahedral tilting drives the structural transitions at high temperatures and strong acoustic attenuation through the temperature interval $\sim 300-500 \mathrm{~K}$, observed by resonant ultrasound spectroscopy from a polycrystalline sample, is consistent with pinning of ferroelastic twin walls by point defects. Below room temperature, stiffening of the shear modulus by up to $\sim 40 \%$ can be understood in terms of biquadratic coupling of the ferromagnetic order parameter with strain. Acoustic attenuation with Debye-like patterns of loss in the temperature interval $\sim 150-280 \mathrm{~K}$ yielded activation energies and relaxation times which match up with AC magnetic and dielectric spectroscopy data reported previously in the literature. The dynamic loss mechanism, perhaps related to hopping of electrons between $\mathrm{Ni}^{2+}$ and $\mathrm{Mn}^{4+}$, is potentially multiferroic, therefore. In addition to the possibilities for tailoring the intrinsic properties of $\mathrm{La}_{2} \mathrm{NiMnO}_{6}$ by controlling oxygen content, $B$-site order or by choice of substrate for imposing a strain on thin films, it should be possible also to engineer extrinsic properties which would respond to applied electric, magnetic, and stress fields.
\end{abstract}

DOI: 10.1103/PhysRevB.100.014304

\section{INTRODUCTION}

Double perovskites $\left(A_{2} B^{\prime} B^{\prime \prime} X_{6}\right)$ exhibit various fundamentally interesting physical and chemical properties, which have been extensively studied in materials science and condensedmatter physics [1-3]. These arise largely as a consequence of the choice of $B$-site cations, leading to a wide range of possibilities for potential device applications [1]. In this context, $\mathrm{La}_{2} \mathrm{NiMnO}_{6}$ (LNMO) is an example of a double perovskite showing magnetoresistance, magnetodielectric, and magnetocapacitance effects near room temperature [4-7]. For example, it can be used in commercially available solidstate thermoelectric (Peltier) coolers [5]. It also has potential application in solar cells because it has been found to display long carrier lifetimes $(\sim 0.1 \mathrm{~ms})$ and robust photovoltaic performance. Further advantages are that it is eco-friendly, nontoxic, cheap, and structurally stable in oxygen and ultraviolet light $[8,9]$.

LNMO has $\mathrm{Ni}^{2+}\left(d^{8}: t_{2 g}^{6} e_{g}^{2}, S=2 / 2\right)$ and $\mathrm{Mn}^{4+}\left(d^{3}\right.$ : $t_{2 g}^{3} e_{g}^{0}, S=3 / 2$ ) ions ordered between crystallographic $B^{\prime}$ and $B^{\prime \prime}$ "sites of the double perovskite structure, and undergoes a paramagnetic-ferromagnetic transition at $\sim 280 \mathrm{~K}$ [5]. The structure of bulk and thin film LNMO is monoclinic $\left(P 2_{1} / n\right)$ at low temperatures. It transforms to rhombohedral $(R \overline{3})$ at high temperature but the two structures coexist over a wide temperature range, including room temperature [5,10-12]. Based on Kanamori-Goodenough rules [13], the ferromagnetic ordering is due to the presence of $180^{\circ} \mathrm{Ni}^{2+}-\mathrm{O}-\mathrm{Mn}^{4+}$ superexchange bonding between an empty $\mathrm{Mn}^{4+} e_{g}$ orbital and the half-filled $d$ orbital of an adjacent $\mathrm{Ni}^{2+}$ ion [14]. Deviations of the Ni-O-Mn bond angle from $180^{\circ}$ do not appear to affect this fundamental aspect of the magnetic behavior [5].

As well as being ferromagnetic near room temperature, LNMO is in principle ferroelastic, giving it two of the ferroic properties that make up single-platform multiferroics. Lowering of the symmetry from the ordered parent cubic $(F m \overline{3} m)$ structure is due to octahedral tilting transitions which occur as a consequence of the small size of $\mathrm{La}^{3+}$ in comparison with the sizes of $\mathrm{NiO}_{6}$ and $\mathrm{MnO}_{6}$ octahedra. Thus, LNMO falls in a class of double perovskites which has close analogies, for example, with $\mathrm{Sr}_{2} \mathrm{FeMoO}_{6}(\mathrm{SFMO}), \mathrm{La}_{2} \mathrm{CoMoO}_{6}$, and $\mathrm{La}_{2} \mathrm{FeMoO}_{6}$ (LFMO). In general, a key issue for the properties and structural evolution of these systems is the strength of structural and magnetic order parameter coupling with strain since this affects not only the strength and form of coupling between the order parameters but also the dynamics and interactions of their transformation microstructures. Analysis of strain/order parameter coupling associated with the improper ferroelastic transition at $\sim 420 \mathrm{~K}$ in SFMO has shown that the ferromagnetic order parameter couples only with volume strain [15]. It exhibits weak magneto-structural coupling, with very closely similar structural and Curie temperatures, but the magnetic properties, electronic properties, microstructures, Curie temperature, and structural transition temperature can still be tuned by the choice of strain imposed from a substrate [16-20]. The phase stability, magnetic, dielectric, and 
electronic properties of LNMO thin films can also be modified by the substrate $[4,11,21-30]$, to the extent that they can be engineered to become ferroelectric [14].

Given the importance of strain relaxation effects associated with multiple phase transitions, it is surprising that these have not been investigated for LNMO in relation to its ferroic/multiferroic properties near room temperature. Although spin-phonon coupling has been observed by Raman spectrum [31], there appear to be no previous studies of either magnetolastic coupling or the influence of ferroelastic twin walls. The primary objective of the present study was to fill this gap by investigating static and dynamic aspects of strain relaxation associated with the magnetic transition using resonant ultrasound spectroscopy (RUS). RUS data obtained from a polycrystalline sample have been used, firstly, to characterize $\sim 40 \%$ stiffening of the shear modulus associated with the paramagnetic-ferromagnetic transition and, secondly, to correlate acoustic loss dynamics with dielectric spectroscopy measurements in a similar temperature/frequency range and $\mathrm{AC}$ magnetic data at lower measuring frequencies. These correlations provide further insights into the pervasive influence of strain coupling on both intrinsic and extrinsic effects at phase transitions in double perovskites [15,32] across wide intervals of temperature. The starting point is a brief review of the known structure-property relations of LNMO. A formal analysis of strain coupling from the perspective of symmetry is given in an appendix.

\section{VARIABILITY IN STRUCTURE-PROPERTY RELATIONS}

Bulk samples of $\mathrm{La}_{2} \mathrm{NiMnO}_{6}$ typically contain a mixture of monoclinic $\left(P 2_{1} / n\right)$ and rhombohedral $(R \overline{3})$ crystals with a range of degrees of $B$-site order at room temperature $[5,12,33,34]$. The proportion of the rhombohedral phase varies between $\sim 25$ and $\sim 70 \%$ [10,33-39], and it appears that increasing oxygen fugacity favors a higher proportion of the monoclinic structure [37].

The expected transition sequence with decreasing temperature is $F m \overline{3} m \rightarrow R \overline{3} \rightarrow P 2_{1} / n$ due to octahedral tilting which is exactly analogous to the sequence $P m \overline{3} m \rightarrow R \overline{3} c \rightarrow P n m a$ of many single $\left(\mathrm{ABO}_{3}\right)$ perovskites [40,41]. Order parameters for the tilting transitions are $X_{3}^{+}$and $\Gamma_{4}^{+}$with respect to the parent $F m \overline{3} m$ structure, as opposed to $\mathrm{M}_{3}^{+}$and $\mathrm{R}_{4}^{+}$with respect to the parent $P m \overline{3} m$ structure. The proportion of the monoclinic phase has been reported to be $100 \%$ at $3.5 \mathrm{~K}$ [5]. An anomaly in the heat capacity at $\sim 648 \mathrm{~K}$ reported by Bull et al. [12] is believed to mark the temperature of the first-order transition between rhombohedral and monoclinic structures, consistent with the disappearance of the monoclinic structure from diffraction patterns collected at temperatures of $673 \mathrm{~K}$ and above [37]. The structure remains rhombohedral up to at least $1073 \mathrm{~K}$, the highest temperature so far reported [37]. Analysis of the lattice parameter data of Sayed et al. [37] in terms of formal strains and their coupling with order parameters for ferromagnetic ordering and octahedral tilting shows that the cubic-rhombohedral transition is likely to be second order in character at an extrapolated transition temperature of $\sim 1880 \mathrm{~K}$ [39]. Evolution of the shear strain $e_{4}$ indicates that coupling between the tilt order parameters and the ferromagnetic order parameter is unfavorable in the rhombohedral structure [Appendix, Fig. 9(b)].

It is well understood that the stoichiometry of bulk LNMO samples can show substantial deviations from ideal stoichiometry, which may be expressed as $\mathrm{La}_{2} \mathrm{MnNiO}_{6+} \delta$, with the understanding that the apparent excess oxygen is achieved by the introduction of cation vacancies $[10,33,36,42]$. Reported values of $\delta$ are predominantly in the range $0.05-0.15[10,33,36]$, implying that the proportion of vacant cation sites could be $\sim 10 \%$. Such variations are not invariably present, however, as Joseph Joly et al. [35] determined that their samples produced by both high and low-temperature routes were stoichiometric within experimental uncertainty. The possibility of oxygen vacancies has also been suggested on the basis of a large reduction in the value of the dielectric constant of samples annealed in oxygen in comparison with those which had been annealed in air or nitrogen [37].

A further factor which depends on synthesis conditions is the balance of the reaction $\mathrm{Ni}^{2+}+\mathrm{Mn}^{4+} \leftrightarrow \mathrm{Ni}^{3+}+\mathrm{Mn}^{3+}$ [10]. Aside from the influence on magnetic properties, this is important because $\mathrm{Mn}^{3+}$ is Jahn-Teller active and can therefore introduce additional distortions locally in the structure. Measurements by x-ray photoluminescence spectroscopy (XPS) have revealed the presence of $\mathrm{Mn}^{3+}$ in some samples and charge balancing requires the presence also of $\mathrm{Ni}^{3+}$. For example, up to $\sim 30 \%$ of the $\mathrm{Mn}$ in samples prepared by a conventional high-temperature synthesis in air has been found to be $\mathrm{Mn}^{3+}$ [34]. Sayed et al. [37] reported the presence of $\mathrm{Ni}^{2+}, \mathrm{Ni}^{3+}, \mathrm{Mn}^{3+}$, and $\mathrm{Mn}^{4+}$ in their sample annealed in air, and Lan et al. [9] and Joseph Joly et al. [35] reported $\mathrm{Mn}^{3+}$ in samples produced by a low-temperature synthesis method. In the case of thin films, at least, it has been proposed that the charge disproportion reaction favors $\mathrm{Mn}^{3+}+\mathrm{Ni}^{3+}$ with decreasing oxygen fugacity [43]. There may not be such consistency in bulk samples, however, as Choudhury et al. [6] found only $\mathrm{Ni}^{2+}$ and $\mathrm{Mn}^{3+}$ in their sample, which is presumed to have been heat treated in air, and Yang et al. [39] had $\mathrm{Mn}^{3+}$ in a sample produced by annealing in oxygen.

Bulk samples display two distinctly different patterns of dielectric properties. One is of a frequency-dependent Debyelike dielectric loss $(\tan \delta)$ peak in the temperature range $\sim 130 \mathrm{~K}(4 \mathrm{kHz})-\sim 210 \mathrm{~K}(1 \mathrm{MHz})$, and the second is a further steep increase at higher temperatures $[6,7,44]$. These are associated with variations of the dielectric constant, $\varepsilon^{6}$, from values on the order of $\sim 100$ or less below $\sim 150 \mathrm{~K}$ up to at least $\sim 4000[6]$ or $\sim 20000[7,44]$ at $300 \mathrm{~K}$. Activation energies in the vicinity of $\sim 0.15-0.2 \mathrm{eV}$ extracted for the loss process were interpreted as being consistent with a mechanism involving asymmetric hopping of charge carriers between $\mathrm{Ni}^{2+}$ and $\mathrm{Mn}^{4+}$ in the applied electric field. The sample of Lin et al. [44] had been sintered in air at $1523 \mathrm{~K}$ and, although this is not specified, the samples of Choudhury et al. [6] and Chandrasekhar et al. [7] are presumed also to have been synthesized in air. Sayed et al. [37] reported similarly high values of $\varepsilon^{\prime}$ in the same temperature interval, although without the discrete peaks in $\tan \delta$, for a sample synthesized in air at $1523 \mathrm{~K}$ followed by annealing in air at $1123 \mathrm{~K}$. However, following annealing at $1123 \mathrm{~K}$ in oxygen, the highest values of $\varepsilon^{\prime}$ reduced to $\sim 200$ and the tan $\delta$ values dropped by nearly 

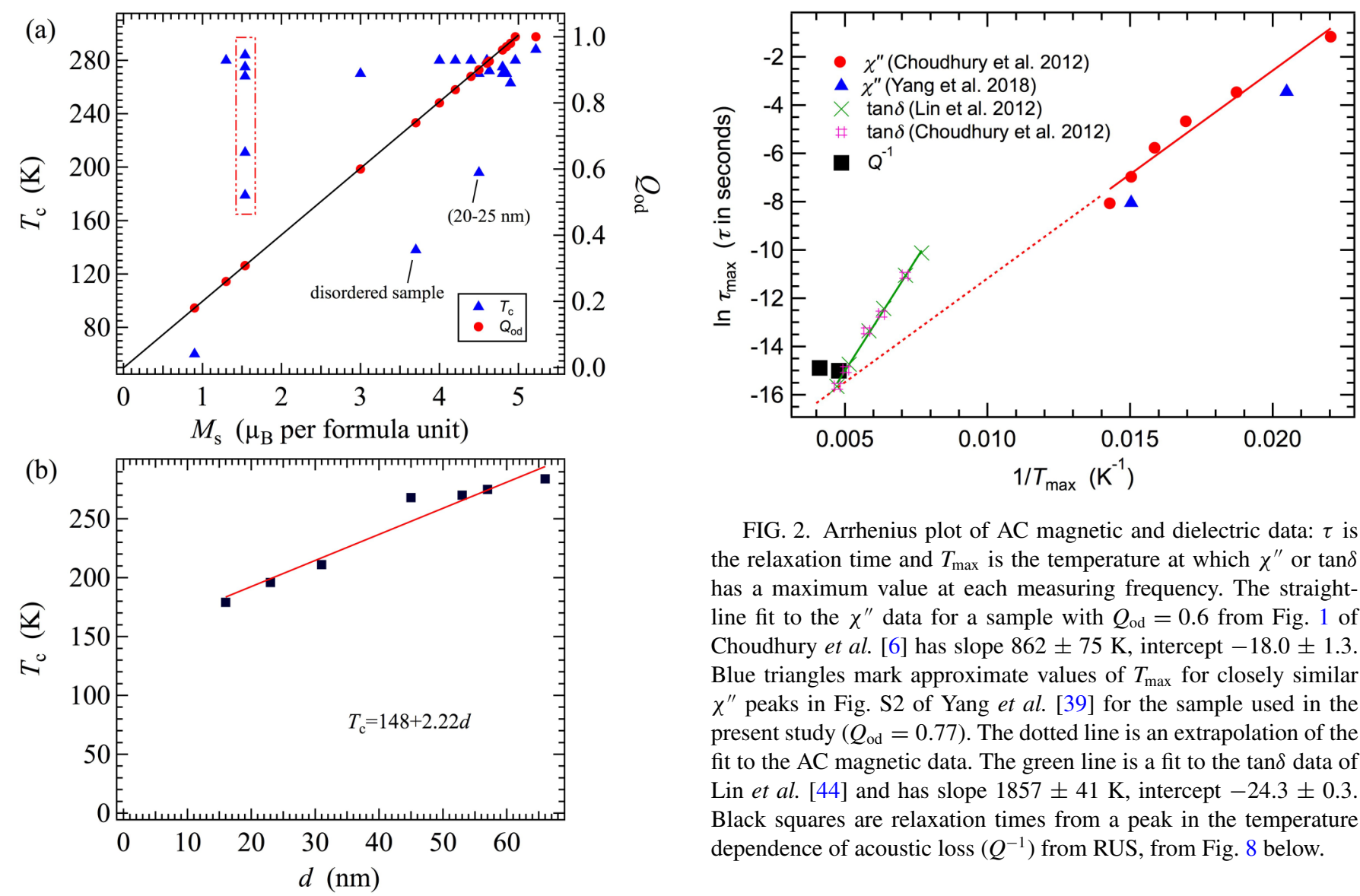

FIG. 2. Arrhenius plot of AC magnetic and dielectric data: $\tau$ is the relaxation time and $T_{\max }$ is the temperature at which $\chi^{\prime \prime}$ or $\tan \delta$ has a maximum value at each measuring frequency. The straightline fit to the $\chi^{\prime \prime}$ data for a sample with $Q_{\text {od }}=0.6$ from Fig. 1 of Choudhury et al. [6] has slope $862 \pm 75 \mathrm{~K}$, intercept $-18.0 \pm 1.3$. Blue triangles mark approximate values of $T_{\max }$ for closely similar $\chi^{\prime \prime}$ peaks in Fig. S2 of Yang et al. [39] for the sample used in the present study $\left(Q_{\mathrm{od}}=0.77\right)$. The dotted line is an extrapolation of the fit to the AC magnetic data. The green line is a fit to the tan $\delta$ data of Lin et al. [44] and has slope $1857 \pm 41 \mathrm{~K}$, intercept $-24.3 \pm 0.3$. Black squares are relaxation times from a peak in the temperature dependence of acoustic loss $\left(Q^{-1}\right)$ from RUS, from Fig. 8 below.

FIG. 1. (a) Summary of literature data for the magnetic phase transition temperature, $T_{\mathrm{c}}$, degree of $B$-site cation order, $Q_{\text {od }}$, and saturation magnetization measured at low temperature, $M_{\mathrm{s}}$, of film and bulk samples $[4,10,11,13-31]$. The straight line is a fit to the data with values of $M_{\mathrm{s}}$ between 0.9 and 4.96 and corresponds to $M_{\mathrm{s}} / M_{\text {sat }}=Q_{\mathrm{od}}$ if the value of $M_{\text {sat }}$ is taken to be 5.0. (b) Summary of literature data for $T_{\mathrm{c}}$ and grain size, $d$, of nanoscale particle samples $[26,29,30]$.

two orders of magnitude. It was concluded that the relaxorlike behavior, with high dielectric constant and high loss, must arise predominantly from the response of oxygen vacancies. Kumar et al. [38] have also reported relatively high values of $\varepsilon^{\prime}$, up to $\sim 2000$, for a sample which had been annealed at $1400{ }^{\circ} \mathrm{C}$, presumably in air. Additional changes of dielectric properties occur in response to an applied magnetic field [5-7] but equivalent measurements on bulk samples prepared by annealing in oxygen do not yet appear to have been reported in the literature.

The theoretical saturation magnetization value is $M_{\text {sat }}=$ $5 \mu_{\mathrm{B}}$ per formula unit, while experimentally determined saturation values, $M_{\mathrm{s}}$, are in the range $0.9-5.2 \mu_{\mathrm{B}}[5-7,11,12,14$, 21-26,31,37,44-51]. It has been shown that $\mathrm{Ni} / \mathrm{Mn}$ ordering is the dominant factor in determining the value of $M_{\mathrm{s}}$ in a given sample [26]. A compilation of data from the literature [Fig. 1(a)] confirms that the relationship with the $B$-site cation order parameter, $Q_{\mathrm{od}}$, is $M_{\mathrm{s}} / M_{\mathrm{sat}}=Q_{\mathrm{od}}$, where $Q_{\mathrm{od}}$ has been defined as $\left(1-2 x_{\mathrm{AS}}\right)$ and $x_{\mathrm{AS}}$ is the proportion of $\mathrm{Ni}$ on the $\mathrm{Mn}$ site and of $\mathrm{Mn}$ on the $\mathrm{Ni}$ site. On the other hand, the

Curie temperature, $T_{\mathrm{c}}$, remains close to $280 \mathrm{~K}$ more or less independently of $M_{\mathrm{s}}$ and $Q_{\mathrm{od}}$ when the grain size is larger than $60 \mathrm{~nm}$ [Fig. 1(a)]. Lower values of $T_{\mathrm{c}}$ framed by the red dotted lines in Fig. 1(a) are due to reducing particle size [47], d. Data from the literature $[47,49,50]$ compiled in Fig. 1(b) show the relationship between $T_{\mathrm{c}}$ and $d$ explicitly as $T_{\mathrm{c}}=$ $148+2.22 d \mathrm{~K}$, for $d<60 \mathrm{~nm}$. It should be noted that the individual particles may consist of one or more crystals, and that the crystals may be separated by large angle boundaries and may have interfaces that are amorphous or crystalline.

The influence of $\mathrm{Ni} / \mathrm{Mn}$ disorder on magnetic properties could also result in the development of a proposed reentrant spin glass at low temperatures, due to magnetic frustration as the proportion of antiferromagnetic $\mathrm{Ni}^{2+}-\mathrm{O}_{-} \mathrm{Ni}^{2+}$ and $\mathrm{Mn}^{4+}$ $\mathrm{O}-\mathrm{Mn}^{4+}$ interactions increases. Evidence for possible glassy freezing of spins has been provided by a frequency-dependent peak in the imaginary component, $\chi^{\prime \prime}$, of the response to an $\mathrm{AC}$ magnetic field in the vicinity of $50 \mathrm{~K}[6,33,38,39]$, and by aging/memory effects at low temperatures arising during interrupted heating sequences [6]. However, Blasco et al. [33] found no anomalies in structural parameters or magnetic moments in refinements of neutron powder-diffraction data and, on this basis, concluded that a reentrant transition from a ferromagnetic phase to a spin glass does not occur. From the data of Choudhury et al. [6], the temperature at which there is a peak in $\chi^{\prime \prime}$ varies between $\sim 45$ and $\sim 70 \mathrm{~K}$ for the frequency range $\sim 1-500 \mathrm{~Hz}$. These results are reproduced in Fig. 2, to show that they may be approximated by the Arrhenius relationship, $\tau=\tau_{\mathrm{o}} \exp \left(E_{\mathrm{a}} / \mathrm{R} T_{\max }\right)$, where $\tau$ is the relaxation time, $\tau_{\mathrm{o}}$ is a constant, $E_{\mathrm{a}}$ is an activation energy, 
$\mathrm{R}$ is the gas constant, and $T_{\max }$ is the temperature where $\chi^{\prime \prime}$ has its maximum value for a given frequency. The peak in $\chi^{\prime \prime}$ is assumed to correspond to the point at which $\omega \tau=1$, where the angular frequency, $\omega$, and the measuring frequency, $f$, are related by $\omega=2 \pi f$. The straight-line fit to the data has $\tau_{\mathrm{o}}=2.5 \times 10^{-9} \mathrm{~s}$ and $E_{\mathrm{a}} / \mathrm{R}=862 \pm 75 \mathrm{~K}\left(E_{\mathrm{a}} \sim 0.07 \pm\right.$ $0.01 \mathrm{eV})$. This activation energy is smaller than values in the range $\sim 0.15-0.2 \mathrm{eV}$ reported from dielectric measurements. A direct comparison is provided in Fig. 2 by the addition of data for the temperatures at which tan $\delta$ has a maximum, from Lin et al. [44] and Choudhury et al. [6]. They define a linear trend with $\tau_{\mathrm{o}}=2.8 \times 10^{-11} \mathrm{~s}$ and $E_{\mathrm{a}} / \mathrm{R}=1857 \pm$ $41 \mathrm{~K}\left(E_{\mathrm{a}} \sim 0.16 \pm 0.01 \mathrm{eV}\right)$.

\section{EXPERIMENTAL METHODS AND SAMPLE CHARACTERIZATION}

\section{A. Synthesis}

The sample of LNMO used in the present study has previously been described in the Supplemental Material of Yang et al. [39]. Reagent-grade $\mathrm{La}_{2} \mathrm{O}_{3}(A R), \mathrm{MnO}_{2}(A R)$, and $\mathrm{NiO}(A R)$ were used as starting materials for the conventional solid-state reaction method of synthesis. Stoichiometric amounts of $\mathrm{La}_{2} \mathrm{O}_{3}, \mathrm{MnO}_{2}$, and $\mathrm{NiO}$ were mixed in ethanol for $6 \mathrm{~h}$ in an agate ball mill. After drying at $353 \mathrm{~K}$ for $12 \mathrm{~h}$, the powder was ground and then calcined at $1573 \mathrm{~K}$ for $6 \mathrm{~h}$ in pure oxygen, with an intermediate grinding stage. The resulting powder was pressed uniaxially at $25 \mathrm{MPa}$ to produce a pellet with $10-\mathrm{mm}$ diameter and thickness $\sim 2 \mathrm{~mm}$, which was sintered at $1573 \mathrm{~K}$ in pure oxygen for $6 \mathrm{~h}$. A rectangular parallelepiped with dimensions $5.158 \times 2.663 \times 0.832 \mathrm{~mm}^{3}$ and mass $0.0441 \mathrm{~g}$ was cut from the sintered pellet for RUS measurements. The sample used for magnetic measurements was cut from another part of the same pellet, and had mass $0.0523 \mathrm{~g}$.

\section{B. Sample characterization}

Scanning electron microscope images of the surface of the ceramic pellet have been given in the Supplemental Material of Yang et al. [39]. They show a porous microstructure containing individual, angular grains with sizes typically in the range 100-500 nm, i.e., well above the range of $d$ values in Fig. 1(b) at which $T_{\mathrm{c}}$ reduces below $\sim 280 \mathrm{~K}$. Elemental distribution maps of $\mathrm{La}, \mathrm{Ni}, \mathrm{Mn}$, and $\mathrm{O}$ obtained with a Bruker Nano $\mathrm{GmbH}$ energy-dispersive $\mathrm{x}$-ray spectrometer showed $\mathrm{La}, \mathrm{Ni}, \mathrm{Mn}$, and $\mathrm{O}$ to be distributed homogeneously in the majority of grains. Ni-rich regions were attributed to $\mathrm{NiO}$ and La-rich regions to the possible presence of amorphous $\mathrm{La}_{2} \mathrm{O}_{3}$. Individual point analyses of perovskite grains gave $\mathrm{La}, \mathrm{Ni}$, $\mathrm{Mn}$, and $\mathrm{O}$ in approximate proportions $2: 1: 1: 6.5$. This is by no means an accurate determination of the oxygen content but it is consistent with data from the literature which imply an oxygen excess and, hence, the presence of cation vacancies [10]. Fitting of the Mn $2 p$ peaks in XPS spectra (Fig. S4 of Yang et al. [39]) yielded an estimate of the proportion of $\mathrm{Mn}$ with charge $3+$ as $8 \%$.

Rietveld refinement of powder x-ray-diffraction data indicated that three crystalline phases were present at room temperature: $62.6 \%$ rhombohedral $(R \overline{3})$ perovskite, $35.7 \%$ monoclinic $\left(P 2_{1} / n\right)$ perovskite, and $1.7 \% \mathrm{NiO}$ [39]. Lattice parameters determined for the monoclinic phase were $a=5.4403(5) \AA, \quad b=5.4759(8) \AA, c=7.7379(5) \AA, \quad \beta=$ $88.926(7)^{\circ}$, and for the rhombohedral phase $a=5.4751(3) \AA$, $\alpha=60.259(5)^{\circ}$. Because Ni and Mn have similar scattering factors, it is not possible to determine the $B$-site occupancies from powder x-ray-diffraction data. However, the value of $M_{\mathrm{s}}$ obtained from DC magnetic measurements at $2 \mathrm{~K}$ was $3.87 \mu_{\mathrm{B}}$ per f.u. [39] which, from the correlation in Fig. 1(a), implies $Q_{\text {od }}=0.77$.

DC magnetic susceptibility data collected as a function of temperature between 2 and $400 \mathrm{~K}$ in a 50 Oe field, using a Quantum Design MPMS superconducting quantum interference device magnetometer, were consistent with a single paramagnetic-ferromagnetic transition at $T_{\mathrm{c}} \approx 285 \mathrm{~K}$ [39]. The coercivity, $H_{\mathrm{c}}$, and remnant magnetization, $M_{\mathrm{r}}$, at $2 \mathrm{~K}$ from measurement of a magnetic hysteresis loop to $+/-7 \mathrm{~T}$ were $23.5 \mathrm{mT}$ and $0.83 \mu_{\mathrm{B}}$ per f.u., respectively [39]. AC magnetic measurements made at 5 and $500 \mathrm{~Hz}$ contained peaks in $\chi^{\prime \prime}$ at $\sim 49$ and $\sim 67 \mathrm{~K}$, respectively. These were closely similar in form to those reported in Choudhury et al. [6] and plot close to the trend shown in Fig. 2. A peak in $\chi^{\prime \prime}$ occurs just below the magnetic transition point (inset in Fig. 1(d) of Yang et al. [39]), as seen also in the data of Choudhury et al. [6].

Dielectric spectra have not been collected from the present sample but, from comparison with the results of Sayed et al. [37] for the influence of oxygenation, they are not expected to have any dielectric loss peaks below room temperature. Increasing dielectric loss with increasing temperature above $\sim 220 \mathrm{~K}$ at measuring frequencies of $1 \mathrm{kHz}-1 \mathrm{MHz}$ would be expected, however (Fig. 11(b) of Sayed et al. [37]).

\section{Raman spectroscopy}

Raman spectra were collected using a LABRAM-HR Evolution confocal laser micro-Raman spectrometer in backscattering geometry, using the 514-nm line of an Ar-ion laser with spectral resolution of $0.5 \mathrm{~cm}^{-1}$. The spectrometer was equipped with a liquid-nitrogen-cooled charge-coupleddevice detector and heating stage. Phonon frequencies and widths at half maximum height were determined using a Lorentzian fit function for the stretching mode, the antistretching or/and bending modes, and two-phonon scattering $\left(\sim 1100-1500 \mathrm{~cm}^{-1}\right)$ peaks via the software package IGOR PRO 6.37 (WaveMetrics).

\section{Resonant ultrasound spectroscopy}

The RUS method for measuring elastic and anelastic properties of samples with dimensions of up to a few millimeters is explained in detail elsewhere in the literature [52-57]. The basic principle is that acoustic resonances of a millimeter-sized object, excited by one piezoelectric transducer and detected by a second, have frequencies, $f$, which depend on its elastic constants. Most resonance modes involve predominantly shearing motions so that, for a polycrystalline sample, changes in $f^{2}$ from individual resonance peaks yield information on variations of the shear modulus. Acoustic loss is measured through the influence on peak widths and is expressed in terms of the 
inverse mechanical quality factor, $Q^{-1}$. In RUS experiments, $Q^{-1}$ is usually taken to be represented by $\Delta f / f$, where $\Delta f$ is the peak width at half maximum height.

Details of the RUS instruments used in the present study are also given elsewhere [53,54]. For temperatures in the range $\sim 5-305 \mathrm{~K}$, the LNMO parallelepiped was placed with opposite faces in direct contact with the two piezoelectric transducers at the end of a stick which was lowered into a helium flow cryostat [58]. Spectra were collected with Dynamic Resonance System "modulus II" electronics. For data collection at temperatures above room temperature, the sample was placed across a pair of corners between the tips of two horizontal alumina buffer rods which were inserted into a resistance furnace [59]. In this setup the transducers are situated on the ends of the buffer rods, outside the furnace, and Stanford electronics are used to collect the spectra [57]. Individual spectra containing 65000 data points in the frequency range $100-1200 \mathrm{kHz}$ (low T) or $50-1200 \mathrm{kHz}$ (high T) were obtained in automated heating and cooling sequences, with a dwell time of 20 min to allow for thermal equilibration before initiating data collection at each set point. Values of $f$ and $\Delta f$ were determined by fitting an asymmetric Lorentzian function to selected peaks in the resulting spectra, using the software package IGOR PRO 6.37 (WaveMetrics).

\section{RESULTS}

\section{A. Raman spectroscopy}

At room temperature, the Raman spectrum of the bulk sample was qualitatively similar to those shown previously in the literature for LNMO and related double perovskites $[34,38,60,61]$. Figure 3(a) includes primary spectra collected in the temperature range $80-650 \mathrm{~K}$, from which it is clear that the dominant features are strong peaks at $\sim 640$ and $\sim 530 \mathrm{~cm}^{-1}$ [Fig. 3(b)]. The mode at $640 \mathrm{~cm}^{-1}$ is due to stretching ("breathing") vibrations of the $(\mathrm{Ni}, \mathrm{Mn}) \mathrm{O}_{6}$ octahedra, while the $530-\mathrm{cm}^{-1}$ mode involves antisymmetric stretching and/or bending $[11,62]$. In reality, these two broad peaks are made up of overlapping peaks from the $R \overline{3}$ and $P 2_{1} / n$ structures $[48,61,62]$.

Conventional hard-mode spectroscopy gives the relationship between an order parameter, $q$, changes in frequency, $\Delta\left(\omega^{2}\right)$, and changes in linewidth, $\Delta \Gamma$, of peaks in Raman and IR spectra as $\Delta\left(\omega^{2}\right) \propto \Delta \Gamma \propto A q^{2}+B q^{4}$, with the normal expectation that $B$ is small [63-65]. For small changes in frequency, the variation of $\Delta\left(\omega^{2}\right)$ can be approximated as the variation of $\Delta \omega$ [65]. The relationship between phonon frequencies and magnetization, $\Delta \omega \propto M^{2}$, has also been shown to apply in the case of Raman modes of LNMO by Iliev et al. [11], based on the model of Granado et al. [66] and Lavadière et al. [67]. With these relationships in mind, values of $\omega$ and $\Gamma$ have been obtained by fitting the three main peaks in Fig. 3. The trends of both parameters do show some changes below $T_{\mathrm{c}}$ (Fig. 4) but not quite as expected in comparison with the observations of Barbosa and Paschoal [61] for a polycrystalline sample of $\mathrm{La}_{2} \mathrm{NiMnO}_{6}$ and of Iliev et al. [62] for a thin film of $\mathrm{La}_{2} \mathrm{CoMnO}_{6}$. The difference arises from the fact that, in both of the latter works, it was possible to fit individual peaks within the overlapping groups which make
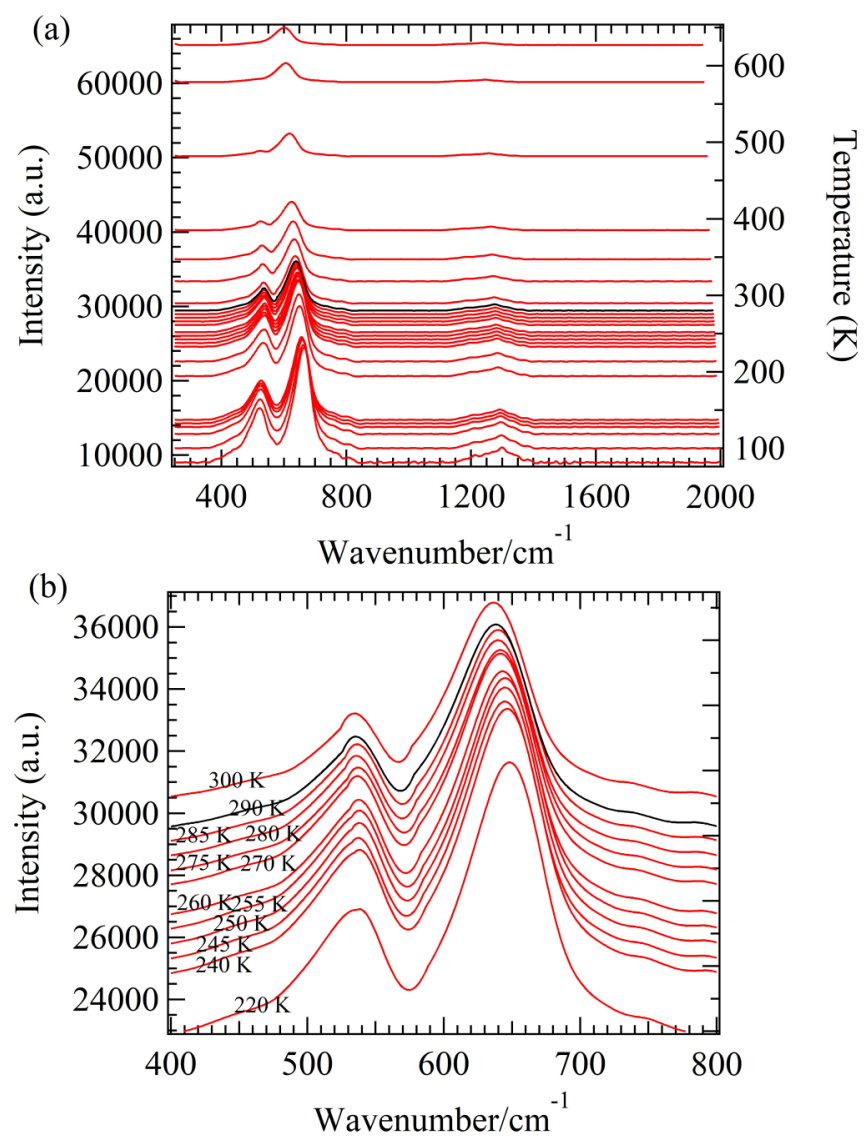

FIG. 3. (a) Raman spectra of $\mathrm{La}_{2} \mathrm{NiMnO}_{6}$ double perovskite in the temperature range $80-650 \mathrm{~K}$, stacked in proportion to the temperatures at which they were collected. (b) Segments of the spectra in the vicinity of the magnetic ordering transition. The spectrum in black was collected at $290 \mathrm{~K}$.

up the peaks at $\sim 530$ and $\sim 670 \mathrm{~cm}^{-1}$. These have widths of $\sim 10-20 \mathrm{~cm}^{-1}[38,48,61]$, rather than $\sim 50-70 \mathrm{~cm}^{-1}$ as shown in Fig. 4. The frequency and peak width variations are strongly influenced by relative shifts in frequency of overlapping peaks rather than from the individual peaks themselves. They nevertheless confirm that there is coupling between phonons and the magnetic order parameter, which probably arises via lattice distortions that accompany the magnetic ordering. Values of $\omega$ and $\Gamma$ for individual phonon modes reduce with falling temperature through $T_{\mathrm{c}}[61]$.

\section{B. Elastic and anelastic behavior}

Illustrative segments of primary RUS spectra collected during heating (red) and cooling (blue) through the temperature interval $\sim 5-305 \mathrm{~K}$ are shown as stacks in Fig. 5, with offsets up the $y$ axis in proportion to the temperatures at which they were collected. The main features are a reduction in the resonance frequencies of all peaks (elastic softening) as the temperature increases and an interval of broadening between $\sim 100$ and $\sim 250$ K. Figure 6 contains segments of spectra collected subsequently in the high-temperature instrument during heating (red) and cooling (blue) between room temperature and $825 \mathrm{~K}$, again stacked in proportion to the temperature at which they were collected. Relatively broad peaks which 


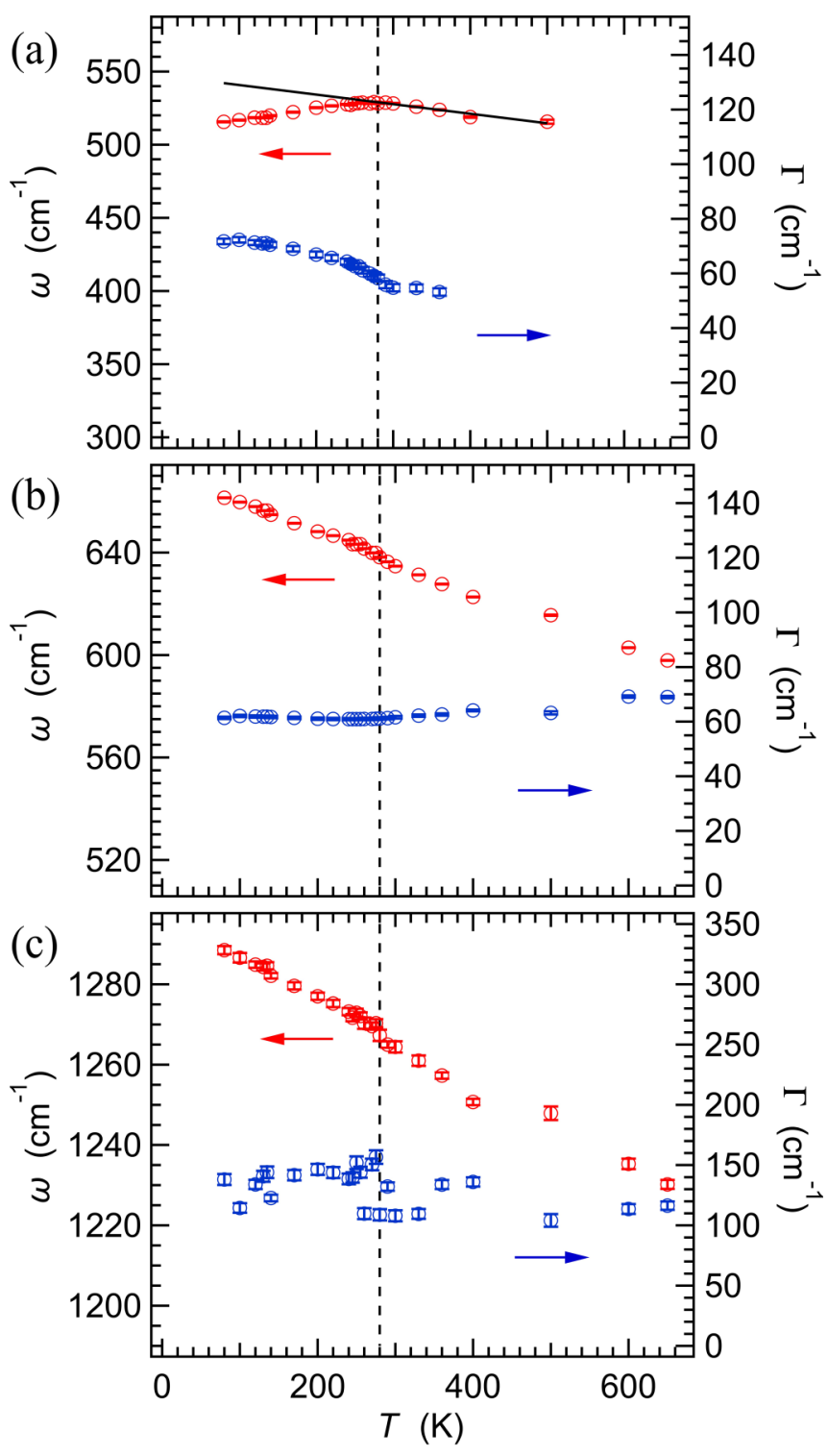

FIG. 4. Variations of phonon frequencies, $\omega$, and individual peak half-widths, $\Gamma$, from the Raman spectra shown in Fig. 3. A dashed black line represents the Curie temperature, shown here as being at $280 \mathrm{~K}$. Error bars are $\pm 1 \sigma$ from peak fitting.

show significant temperature dependence are from the sample, while peaks which show little or no change in frequency with temperature are from the buffer rods. At room temperature and up to $\sim 500 \mathrm{~K}$ in the heating sequence [Fig. 6(a)] there are no obvious resonance peaks from the sample in the frequency range shown, although some of the rod peaks have enhanced amplitudes due to convolution with sample resonances that are too weak to be appear directly in the spectrum. Distinct sample resonances in the higher range of temperatures, such as at $\sim 180-190 \mathrm{kHz}$ above $\sim 500 \mathrm{~K}$, show a weak, nonlinear temperature dependence. In the cooling sequence [Fig. 6(b)] this pattern is reversed, but resonances from the sample reappear below $\sim 450 \mathrm{~K}$.

Figure 7 contains a compilation of $f^{2}$ and $Q^{-1}$ data from individual resonance peaks that could be followed throughout most of the full temperature range over which spectra
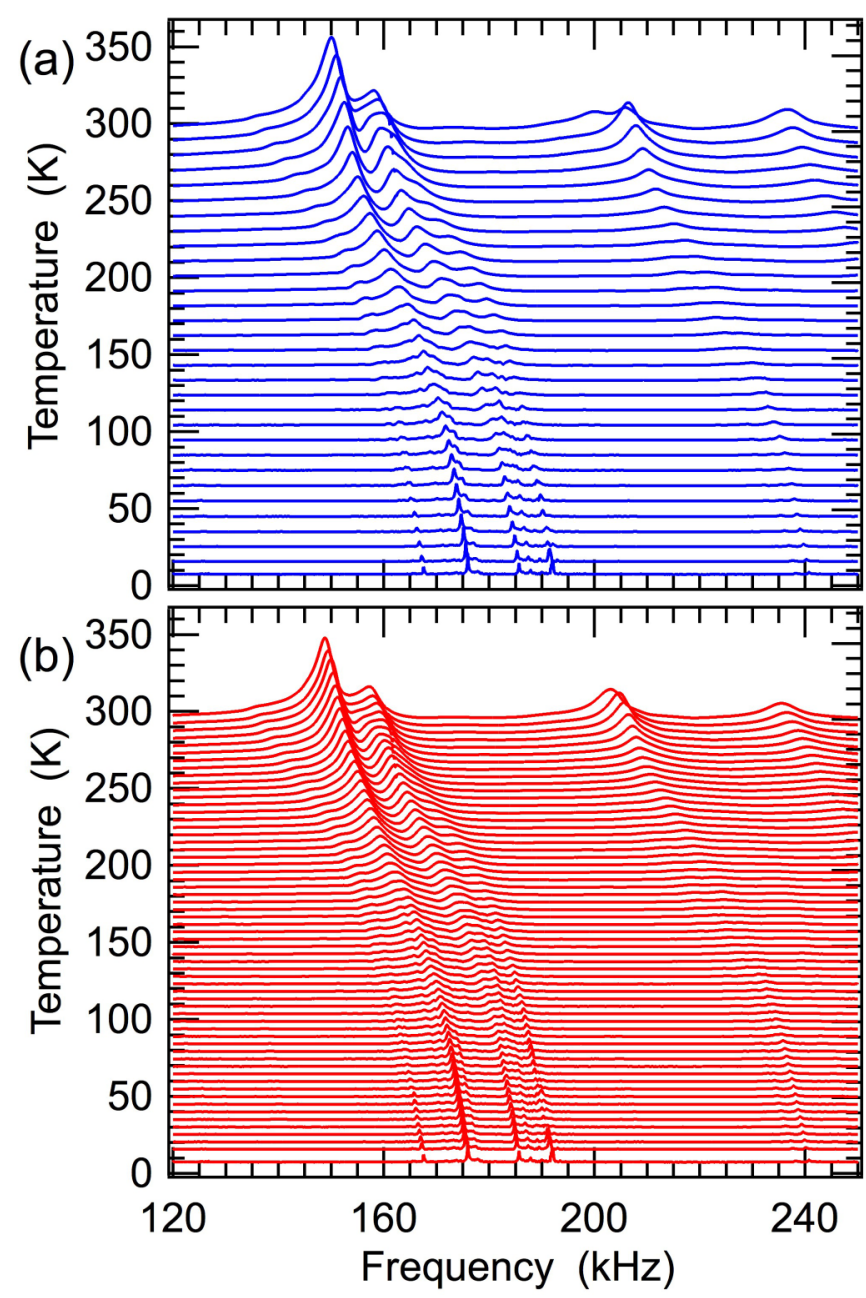

FIG. 5. Segments of RUS spectra for the polycrystalline sample of $\mathrm{La}_{2} \mathrm{NiMnO}_{6}$ from the low-temperature instrument. The left axis is amplitude (volts) but the spectra have been offset in proportion to the temperature at which they were collected and the axis labeled as temperature. There is a distinct interval between $\sim 100$ and $\sim 250 \mathrm{~K}$ where the resonance peaks show marked broadening. (a) Cooling in $10-\mathrm{K}$ steps. (b) Heating in 5-K steps.

were collected. Values of $f^{2}$ from peaks in spectra from the low-temperature instrument have been scaled so that they all overlap, confirming a consistent pattern of softening of the shear modulus with increasing temperature. $f^{2}$ data from the heating sequence of the high-temperature instrument were scaled so that they match the values from the low-temperature instrument at room temperature. There is a small hysteresis between heating and cooling in the hightemperature measurements such that the value of $f^{2}$ at room temperature was $\sim 4 \%$ lower after heating to $825 \mathrm{~K}$ than before. This gives the appearance in Fig. 7 of there being a small discontinuity at room temperature, whereas the data are in fact consistent with a continuous variation through the heating sequence from $\sim 5$ to $\sim 825 \mathrm{~K}$. A small hysteresis in $f^{2}$ values is also evident between $\sim 800$ and $600 \mathrm{~K}$ where values for the cooling sequence show that the sample was slightly stiffer than it had been during heating. A vertical broken line is shown at $280 \mathrm{~K}$ for the approximate position of the magnetic 

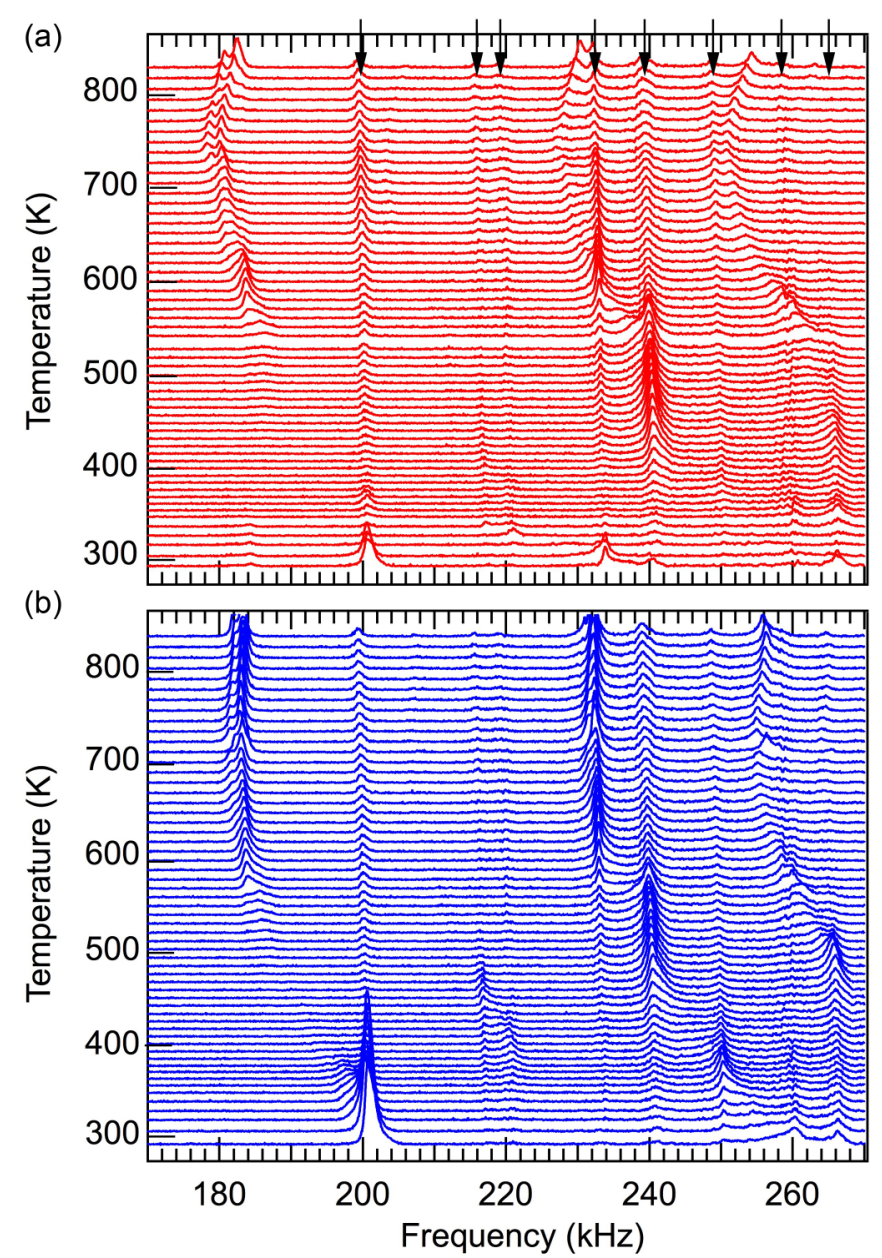

FIG. 6. Segments of RUS spectra from the high-temperature instrument. As in Fig. 5, the spectra have been offset up the $y$ axis in proportion to the temperature at which they were collected and the axis relabeled as temperature. Resonance peaks which do not shift in frequency with changing temperature are from the alumina buffer rods and are indicated by arrows. Peaks which show a clear temperature dependence, such as the peak with frequency variations in the range $180-200 \mathrm{kHz}$, are from the sample. (a) Heating in $\sim 10-15-\mathrm{K}$ steps. (b) Cooling in $\sim 10-15-\mathrm{K}$ steps. In the heating sequence, resonance peaks first appeared at $\sim 500 \mathrm{~K}$. In the cooling sequence, the pattern is reversed except that some weak sample peaks become visible below $\sim 450 \mathrm{~K}$. Resonance peaks from the buffer rods were enhanced where they interacted with broad resonances of the sample which would not otherwise be visible.

ordering temperature. There is no obvious discontinuity at this temperature. Instead, there is an increase in the slope of $f^{2}$ with falling temperature, indicative of an increased stiffening of the shear modulus which amounts to $\sim 40 \%$ between $\sim 300$ and $\sim 5 \mathrm{~K}$. There is no overt anomaly in either $f^{2}$ or $Q^{-1}$ near $650 \mathrm{~K}$, which is the temperature at which an exothermic reaction was reported by Bull et al. [12] and which may have marked the temperature of the hexagonal-monoclinic transition.

Figure 7 also shows a distinctive pattern of acoustic loss in both the heating and cooling sequences. As seen in detail for selected resonance peaks in Fig. $8, Q^{-1}$ is $\sim 0.002$ at $\sim 5 \mathrm{~K}$, increasing to $\sim 0.04$ in the interval $\sim 210-260 \mathrm{~K}$, before

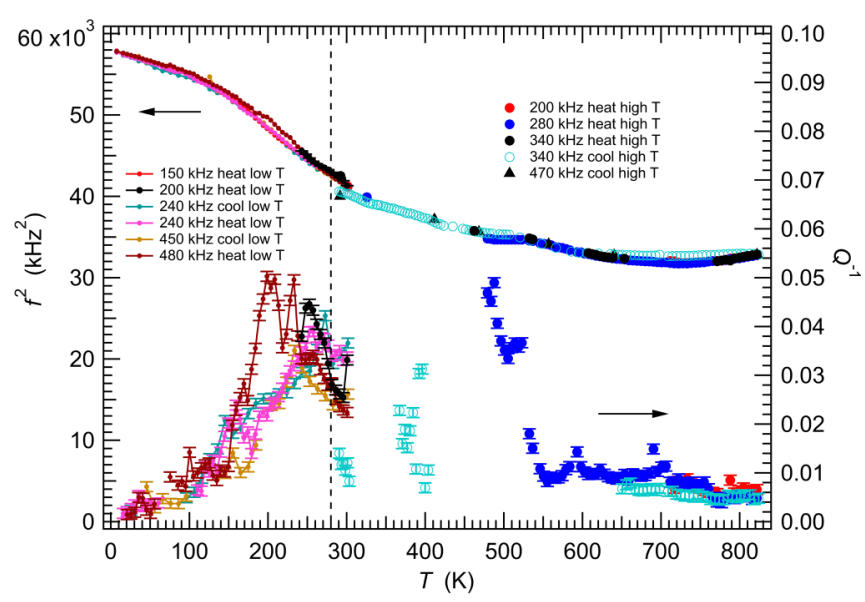

FIG. 7. Variations of the square of the frequency, $f^{2}$, and inverse mechanical quality factor, $Q^{-1}$, for selected resonances from the primary resonance spectra. Labels refer to the approximate frequency at room temperature for which the data are given. $f^{2}$ values from the low- $T$ instrument have been scaled so that they all overlap closely between $\sim 5$ and $300 \mathrm{~K}$. Uncertainties from peak fitting are on the order of or smaller than the symbols for $f^{2}$ and are shown, conservatively, as \pm 0.001 for $Q^{-1}$. The variation of $f^{2}$ appears to be continuous on heating through the magnetic transition, the approximate position of which is indicated by a dashed vertical line at $280 \mathrm{~K} . f^{2}$ values in heating sequences from the high- $T$ instrument have been scaled so that they match the low- $T$ values at room temperature but a slight hysteresis on subsequent cooling results in a difference of $\sim 4 \%$ at $300 \mathrm{~K}$ so that it appears as though there is a discontinuity. Resonance peaks of the sample were not detected directly in the high- $T$ spectra between 300 and $\sim 450 \mathrm{~K}$, although their influence can be seen as an enhancement of resonances from the buffer rods. Sample resonances reappeared in the heating sequence and show steeply reducing values of $Q^{-1}$ in the interval $470-550 \mathrm{~K}$, above which there is a steady baseline. On cooling, sample resonances disappeared at $\sim 650 \mathrm{~K}$ and reappeared below $\sim 400 \mathrm{~K}$, although their low amplitudes resulted in a large scatter of the measured peak widths. With falling temperature below $300 \mathrm{~K}$, values of $Q^{-1}$ have an overall pattern of first increasing and then decreasing to $\sim 0.003$ at $\sim 5 \mathrm{~K}$.

reducing again. There does not appear to be any obvious additional anomaly specifically at the magnetic transition temperature. Sample resonances were not detected directly as peaks in spectra collected during heating from room temperature to $\sim 440 \mathrm{~K}$ because of additional attenuation due to the alumina buffer rods in the high-temperature instrument. The first sample resonance appeared at $\sim 440 \mathrm{~K}$ and was well enough defined to allow fitting above $\sim 480 \mathrm{~K}$. This reduced in width steeply with increasing temperature, reaching a steady baseline of $Q^{-1} \approx 0.05-0.1$ at $\sim 550 \mathrm{~K}$. In the cooling sequence, the same resonance peak (at $\sim 260 \mathrm{kHz}$ ) was not detectable between $\sim 650$ and $\sim 450 \mathrm{~K}$. There is noise in the measured values of $Q^{-1}$ shown near 400 and $300 \mathrm{~K}$ in Fig. 7 but the peak was certainly broad in comparison with its width at higher temperatures. These observations indicate strong attenuation in the interval $\sim 300-500 \mathrm{~K}$ during heating and in the interval $\sim 650-400 \mathrm{~K}$ during cooling. This hysteresis and that seen also in $f^{2}$ values are most likely to have been a consequence of changing stoichiometry resulting 


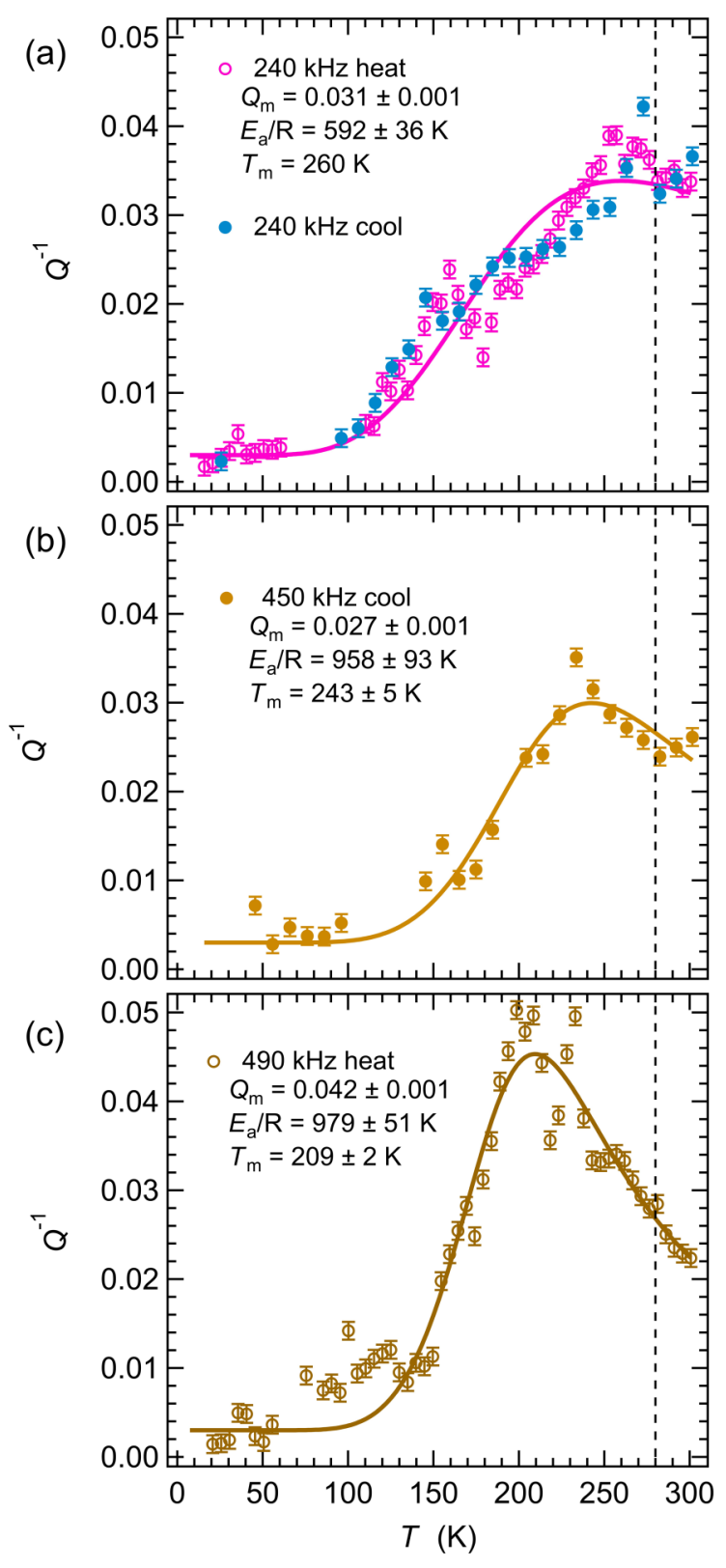

FIG. 8. Variations of $Q^{-1}$ for selected resonances below room temperature. Uncertainties from peak fitting are shown, conservatively, as \pm 0.001 . Solid lines are fits of Eq. (1) to the data, using a constant baseline of $Q^{-1}=0.003$ and with the value of $r_{2}(\beta)$ set at 1 .

from annealing the sample in air at temperatures up to $\sim 825 \mathrm{~K}$ over a total period of a few days during the data collection.

The simplest interpretation of rounded peaks in $Q^{-1}$ as a function of temperature is that they are due to a thermally activated loss mechanism which can be described by the Debye equations. A standard fitting procedure for acoustic data makes use of the expression [15,68-73]

$$
Q^{-1}(T)=Q_{\mathrm{m}}^{-1}\left[\cosh \left\{\frac{E_{a}}{\mathrm{R} r_{2}(\beta)}\left(\frac{1}{T}-\frac{1}{T_{\mathrm{m}}}\right)\right\}\right]^{-1},
$$

where $\mathrm{R}$ is the gas constant, $T_{\mathrm{m}}$ is the temperature at which the maximum value of $Q_{\mathrm{m}}, Q_{\mathrm{m}}^{-1}$, occurs, $E_{\mathrm{a}}$ is an activation energy, and $r_{2}(\beta)$ is a width parameter which describes the spread of relaxation times. Figure 8 shows fits for $Q^{-1}$ from three different resonances with the value of $r_{2}(\beta)$ set at 1 , corresponding to a single relaxation time at each temperature. The curve shown for $240 \mathrm{kHz}$ (heating) was poorly constrained and was assumed to have $T_{\mathrm{m}}=260 \mathrm{~K}$, but the data for resonances with frequencies near 450 and $490 \mathrm{kHz}$ are well represented by this treatment. The activation energies from the latter two peaks under the assumption of $r_{2}(\beta)=1$ are $958 \pm 93$ and $979 \pm 51 \mathrm{~K}\left(E_{\mathrm{a}} \sim 0.08 \pm 0.01 \mathrm{eV}\right)$.

$T_{\mathrm{m}}$ and relaxation time, $\tau=1 / 2 \pi f$, values from the fits for $\sim 450$ and $\sim 490 \mathrm{kHz}$ have been added to Fig. 2 to show that they plot close both to measurements of $\tan \delta$ from dielectric spectroscopy and to a linear (Arrhenius) extrapolation of $\chi^{\prime \prime}$ from AC magnetism. If the acoustic loss arises from the same mechanism as the dielectric loss, the activation energy must be $\sim 0.16 \mathrm{eV}$, giving $r_{2}(\beta) \approx$ and implying a spread of relaxation times. On the other hand, the activation energy for acoustic loss with $r_{2}(\beta)=1$ is indistinguishable from $0.07 \pm 0.01 \mathrm{eV}$ from AC magnetism. Thus the acoustic loss could be related to either or both. The only caveat is that $T_{\mathrm{m}}$ should increase with increasing frequency for a thermally activated process and the $Q^{-1}$ data have $T_{\mathrm{m}}$ decreasing instead (Fig. 8). This might also be due to overlapping contributions from a further loss mechanism, such as critical slowing down, associated with the transition point itself. In this context, it is notable that there is also a peak in $\chi$ " at $\sim 255 \mathrm{~K}$ which does not vary with frequency [6].

\section{DISCUSSION}

On the basis of the strain analysis presented in the Appendix, octahedral transitions in LNMO appear to be typical of the way that the two classical tilt systems of perovskites develop. The cubic-rhombohedral transition is expected to be second order in character with, by extrapolation, a transition temperature of $\sim 1880 \mathrm{~K}$. The second tilt system develops at a first-order transition which has not yet been fully characterized but appears to be in the vicinity of $\sim 650 \mathrm{~K}$. The only unusual feature is the wide temperature interval over which the rhombohedral and monoclinic phases coexist. This may well account for the absence of any obvious acoustic anomalies associated with the rhombohedral-monoclinic transition. Development of the second tilt system (Imma $\rightarrow$ Pnma) in the single perovskites $\mathrm{SrZrO}_{3}$ and $\mathrm{BaCeO}_{3}$ is accompanied by an increase in the shear modulus [74,75]. In $\mathrm{Ca}_{0.7} \mathrm{Sr}_{0.3} \mathrm{TiO}_{3}$ the $I 4 / \mathrm{mcm} \rightarrow$ Pnma transition is barely detectable in the evolution of the shear modulus [76]. For LNMO any increase in shear modulus due to the $R \overline{3} \rightarrow P 2_{1} / n$ transition would be smeared out over a wide temperature interval due to the two-phase coexistence. This stiffening may be reflected in the increase of $f^{2}$ by $\sim 30 \%$ between $650 \mathrm{~K}$ and room temperature for all resonances (Fig. 7), although part of this stiffening must be due to the normal effects of thermal expansion.

Variations in the anelastic properties of LNMO above room temperature are also consistent with typical perovskite behavior in displaying high acoustic loss which can be attributed to the mobility of ferroelastic twin walls. RUS measurements of single crystals of $\mathrm{LaAlO}_{3}$ have shown that high attenuation reduces steeply below $\sim 500 \mathrm{~K}$ where twin walls in the rhombohedral structure become pinned [77]. On the basis of a value 
of $\sim 0.9 \mathrm{eV}$ for the activation energy, the thermally activated pinning mechanism had previously been understood to be due to interaction of the walls with oxygen vacancies [78]. RUS data for a ceramic sample of SFMO show a peak in $Q^{-1}$ between 300 and $400 \mathrm{~K}$ with an estimated activation energy of $\geqslant 0.65 \mathrm{eV}$, consistent with essentially the same pinning process in the rhombohedral phase [15]. Although it has not been possible to extract an activation energy in the case of LNMO because the loss becomes too great, the interval below $\sim 550 \mathrm{~K}$ where attenuation increases steeply is most likely to be due to the same immobilization of ferroelastic twin walls by interaction with defects. Some sensitivity to oxygen content is seen in the upward shift of this freezing interval following the heating cycle up to $825 \mathrm{~K}$ in air of the initially oxygenated sample.

The strain data below $T_{\mathrm{c}}$ show a trend of reducing values of $e_{4}$ in the rhombohedral phase with falling temperature [Fig. 9(b)]. Equation (A14) requires that coupling of shear strain with the magnetic order parameter, $\lambda e_{4} q_{\mathrm{m}}{ }^{2}$, has opposite sign from coupling with the order parameter for parameter for $\Gamma_{4}^{+}$tilting, $\lambda e_{4} q_{\mathrm{t}}{ }^{2}$. It follows that coupling between the two order parameters, $\lambda q_{\mathrm{t}}{ }^{2} q_{\mathrm{m}}{ }^{2}$, is unfavorable, such that increases in magnetic order must cause a reduction of the octahedral tilting. The evolution of $e_{6}$ for the monoclinic structure has the same dependence on the magnetic and tilt order parameters as $e_{4}$ of the rhombohedral structure [Eq. (A10)] and the same reversal in trend below $T_{\mathrm{c}}$ [Fig. 9(b)], indicating that the same unfavorable coupling persists. An immediate consequence of linear-quadratic coupling, $\lambda e q^{2}$, which applies also to volume strains, is that the elastic constants would be expected to show steplike softening below $T_{\mathrm{c}}$, as occurs, for example, at the antiferromagnetic transition in $\mathrm{CoF}_{2}$ [79]. Instead, for LNMO there is a break in slope of $f^{2}$ followed by stiffening of up to $\sim 40 \%$ (Fig. 7), with a form that is closely similar to that previously found below $\sim 160 \mathrm{~K}$ in LFMO (Yang et al., 2019 [80]). The implication is that either the timescale for relaxation of the order parameter in response to a change in strain is large in comparison with the timescale of the applied stress, or some other blocking process occurs. Stiffening or softening proportional to $q^{2}$ will still occur via a biquadratic term, $\lambda e^{2} q^{2}$ which is always allowed, however (see, for example, Carpenter and Salje [81]). The outcome for LNMO is that the stiffening has the same form of variation as $q_{\mathrm{m}}{ }^{2}$ seen for a thin film [11] and as the change in frequency of a Raman mode from a polycrystalline sample [61]. In other words, changes in elastic constants which contribute to the shear modulus scale with $q_{\mathrm{m}}{ }^{2}$, due to coupling of the form $\lambda \mathrm{e}^{2} q_{\mathrm{m}}{ }^{2}$.

The temperatures and relaxation times extracted from peaks in the temperature dependence of $Q^{-1}$ lie close to linear extrapolations of fits to data for peaks in $\chi^{\prime \prime}$ from AC magnetism (Fig. 2 above; Fig. 10 of Yang et al., 2019 [80]). One possibility is that both loss processes are due to the mobility of boundaries between magnetic clusters associated with the reentrant glass transition proposed by Choudhury et al. [6] and Kumar et al. [38]. However, this appears to be ruled out for LNMO in the light of the conclusion of Blasco et al. [33] that results from refinement of structural parameters and magnetic moment are not consistent with a reentrant glass transition. A second possible correlation is that both the magnetic and the acoustic loss behavior are due to the thermally activated pinning of magnetic domain walls, as suggested by Blasco et al. [33]. For this to be the case, it is necessary that there should be both strain contrast and a change in the orientation of magnetic moments across the walls. Ferroelastic twin walls are typically a few unit cells thick (e.g., Chrosch and Salje [82], Hayward et al. [83]) and their mobility in the conditions of low applied stress and $\mathrm{kHz}-\mathrm{MHz}$ frequencies of an RUS experiment is probably by displacement of ledges within the walls [84,85]. Local motion of ledges at a ferroelastic twin wall within or crossing a magnetic domain boundary below the main freezing temperature could give rise to changes in moment because of the coupling between the magnetic and ferroelastic order parameters. The unfavorable coupling between the two would ensure that, because the shear strain and tilt order parameters tend to zero at the center of a ferroelastic twin wall, the local degree of magnetization will be higher within the walls than in the matrix. The resulting motion of ledges could resemble that of local moments with a strain cloud, and the activation energy of $\sim 0.07 \mathrm{eV}$ from Fig. 2 is similar to values in the vicinity of $\sim 0.1 \mathrm{eV}$ discussed for the relaxation behavior of magnetic polarons in $\mathrm{LaCoO}_{3}$ by Zhang et al. [86]. A recent observation of glasslike dynamics of polar domain walls in $\mathrm{SrTiO}_{3}$ [87] shows that aging/memory effects can arise by motion of domain walls in a heterogeneous medium. Similar effects might account for the glassy aging behavior observed by Choudhury et al. [6] in LNMO, with magnetic and charge heterogeneity arising from $\mathrm{Ni} / \mathrm{Mn}$ disorder and/or variations of magnetic moments locally at ferroelastic twin walls.

A third possibility is that the dielectric and acoustic loss mechanisms are essentially the same-hence the overlap of data for maxima in $\tan \delta$ and $Q^{-1}$ shown in Fig. 2. In this case, the pinning/freezing process must involve local changes in both strain and electric dipole, with an activation energy of $\sim 0.16 \mathrm{eV}$. XPS data [39] indicated that the proportion of Mn in the $3+$ state was $\sim 8 \%$ and, since $\mathrm{Mn}^{3+}$ is Jahn-Teller active but $\mathrm{Mn}^{4+}$ is not, transfer of charge between $\mathrm{Ni}^{2+}$ and $\mathrm{Mn}^{4+}$ would be accompanied by some local distortion. The charge transfer would also give rise to changes in local magnetic states, however, which leads to the most interesting possibility of all, namely that the extrinsic loss process involves truly multiferroic responses to externally applied stress, magnetic and electric fields. The dielectric properties are sensitive to the oxygen content of the sample, providing a potential means of tuning these responses. It is worth remembering, also, that LNMO in thin-film form can be induced to become ferroelectric [13]. In order to follow up on these aspects in detail, accurate determinations of the concentrations of $\mathrm{Ni}^{2+}$, $\mathrm{Ni}^{3+}, \mathrm{Mn}^{3+}, \mathrm{Mn}^{4+}$, and vacancies, together with their degree of order, will be required for both bulk and thin-film samples.

\section{CONCLUSION}

LNMO is an example of a multiferroic material which is ferroelastic and ferromagnetic, with significant intrinsic magnetoelastic coupling in bulk samples and the potential to become ferroelectric in thin-film form. Coupling between the structural and magnetic order parameters includes a common shear strain in the rhombohedral structure and appears to be unfavorable. This common strain is seen also in the 
spin-lattice coupling identified by Raman spectroscopy. In addition, LNMO displays significant acoustic, AC magnetic, and dielectric loss mechanisms which have an extrinsic origin. One interesting possibility is that the magnetoelastic loss mechanism is due to interaction between ferromagnetic and ferroelastic domain walls. An alternative but equally interesting possibility is that the dynamics of charge transfer between $\mathrm{Ni}^{2+}$ and $\mathrm{Mn}^{3+}$ in the partially disordered involves changes in local strain, magnetic order, and electric polarization. These coupled properties can, in principle, be manipulated by the choice of imposed strain from a substrate, the degree of $B$-site cation order, and the control of vacancy concentrations by heat treatments under oxidizing or reducing conditions.

\section{ACKNOWLEDGMENTS}

This work was supported financially by the National Natural Science Foundation of China (Grant No. 51702289) and the China Postdoctoral Science Foundation (Grant No.
2016M601963). RUS facilities have been established and maintained in Cambridge through grants from the Natural Environment Research Council and the Engineering and Physical Sciences Research Council of Great Britain to MAC, which are gratefully acknowledged (Grants No. NE/B505738/1, No. NE/F17081/1, and No. EP/I036079/1).

\section{APPENDIX: SYMMETRY AND STRAIN ANALYSIS}

With respect to a parent structure with space group $F m \overline{3} m$, the active representations for in-phase and out-of-phase tilting transitions are $X_{3}^{+}$and $\Gamma_{4}^{+}$(ISOTROPY Software Suite, https://iso.byu.edu/iso/isotropy.php).There is only one possible active representation for ferromagnetic ordering in LNMO and that is $\mathrm{m}_{4}^{+}$. Each of the order parameters has three components, $q_{1}-q_{3}$ for $X_{3}^{+}, q_{4}-q_{6}$ for $\Gamma_{4}^{+}$and $m_{1}-m_{3}$ for $\mathrm{m} \Gamma_{4}^{+}$. ISOTROPY gives the following free-energy expansion, including coupling with strains:

$$
\begin{aligned}
& G=\frac{1}{2} a_{1} \Theta_{\mathrm{s} 1}\left(\operatorname{coth}\left(\frac{\Theta_{\mathrm{s} 1}}{T}\right)-\operatorname{coth}\left(\frac{\Theta_{\mathrm{s} 1}}{T_{\mathrm{s} 1}}\right)\right)\left(q_{1}^{2}+q_{2}^{2}+q_{3}^{2}\right)+\frac{1}{4} b_{1}\left(q_{1}^{2}+q_{2}^{2}+q_{3}^{2}\right)^{2}+\frac{1}{4} b_{1}^{\prime}\left(q_{1}^{4}+q_{2}^{4}+q_{3}^{4}\right) \\
& +\frac{1}{2} a_{2} \Theta_{\mathrm{s} 2}\left(\operatorname{coth}\left(\frac{\Theta_{\mathrm{s} 2}}{T}\right)-\operatorname{coth}\left(\frac{\Theta_{\mathrm{s} 2}}{T_{\mathrm{s} 2}}\right)\right)\left(q_{4}^{2}+q_{5}^{2}+q_{6}^{2}\right)+\frac{1}{4} b_{2}\left(q_{4}^{2}+q_{5}^{2}+q_{6}^{2}\right)^{2}+\frac{1}{4} b_{2}^{\prime}\left(q_{4}^{4}+q_{5}^{4}+q_{6}^{4}\right) \\
& +\frac{1}{2} a_{3} \Theta_{\mathrm{s} 3}\left(\operatorname{coth}\left(\frac{\Theta_{\mathrm{s} 3}}{T}\right)-\operatorname{coth}\left(\frac{\Theta_{\mathrm{s} 3}}{T_{\mathrm{c}}}\right)\right)\left(m_{1}^{2}+m_{2}^{2}+m_{3}^{2}\right)+\frac{1}{4} b_{3}\left(m_{1}^{2}+m_{2}^{2}+m_{3}^{2}\right)^{2}+\frac{1}{4} b_{3}^{\prime}\left(m_{1}^{4}+m_{2}^{4}+m_{3}^{4}\right) \\
& +\lambda_{1} e_{\mathrm{a}}\left(q_{1}^{2}+q_{2}^{2}+q_{3}^{2}\right)+\lambda_{2} e_{\mathrm{a}}\left(q_{4}^{2}+q_{5}^{2}+q_{6}^{2}\right)+\lambda_{3} e_{\mathrm{a}}\left(m_{1}^{2}+m_{2}^{2}+m_{3}^{2}\right)+\lambda_{4}\left[\sqrt{3} e_{\mathrm{o}}\left(q_{2}^{2}-q_{1}^{2}\right)-e_{\mathrm{t}}\left(q_{1}^{2}+q_{2}^{2}-2 q_{3}^{2}\right)\right] \\
& +\lambda_{5}\left[\sqrt{3} e_{\mathrm{o}}\left(q_{4}^{2}-q_{5}^{2}\right)-e_{\mathrm{t}}\left(q_{4}^{2}+q_{5}^{2}-2 q_{6}^{2}\right)\right]+\lambda_{6}\left[\sqrt{3} e_{\mathrm{o}}\left(m_{1}^{2}-m_{2}^{2}\right)-e_{\mathrm{t}}\left(m_{1}^{2}+m_{2}^{2}-2 m_{3}^{2}\right)\right] \\
& +\lambda_{7}\left(e_{4}^{2}+e_{5}^{2}+e_{6}^{2}\right)\left(q_{1}^{2}+q_{2}^{2}+q_{3}^{2}\right)+\lambda_{8}\left(\left(e_{6}^{2}+e_{4}^{2}\right) q_{1}^{2}+\left(e_{6}^{2}+e_{5}^{2}\right) q_{2}^{2}+\left(e_{4}^{2}+e_{5}^{2}\right) q_{3}^{2}\right)+\lambda_{9}\left(e_{4} q_{5} q_{6}+e_{5} q_{4} q_{6}+e_{6} q_{4} q_{5}\right) \\
& +\lambda_{10}\left(q_{1}^{2}\left(e_{4} q_{4}-e_{6} q_{6}\right)+q_{2}^{2}\left(e_{6} q_{6}-e_{5} q_{5}\right)+q_{3}^{2}\left(e_{5} q_{5}-e_{4} q_{4}\right)\right)+\lambda_{11}\left(e_{4} m_{2} m_{3}+e_{5} m_{1} m_{3}+e_{6} m_{1} m_{2}\right) \\
& +\lambda_{X 3+\Gamma 4+}\left(q_{1}^{2}+q_{2}^{2}+q_{3}^{2}\right)\left(q_{4}^{2}+q_{5}^{2}+q_{6}^{2}\right)+\lambda_{X 3+\Gamma 4+}^{\prime}\left(q_{1}^{2}\left(q_{4}^{2}+q_{6}^{2}\right)+q_{2}^{2}\left(q_{5}^{2}+q_{6}^{2}\right)+q_{3}^{2}\left(q_{4}^{2}+q_{5}^{2}\right)\right) \\
& +\lambda_{X 3+\mathrm{m} \Gamma 4+}\left(q_{1}^{2}+q_{2}^{2}+q_{3}^{2}\right)\left(m_{1}^{2}+m_{2}^{2}+m_{3}^{2}\right)+\lambda_{X 3+\mathrm{m} \Gamma 4+}^{\prime}\left(q_{1}^{2}\left(m_{1}^{2}+m_{3}^{2}\right)+q_{2}^{2}\left(m_{2}^{2}+m_{3}^{2}\right)+q_{3}^{2}\left(m_{1}^{2}+m_{2}^{2}\right)\right) \\
& +\lambda_{\Gamma 4+\mathrm{m} \Gamma 4+}\left(q_{4} m_{1}+q_{5} m_{2}+q_{6} m_{3}\right)^{2}+\lambda_{\Gamma 4+\mathrm{m} \Gamma 4+}^{\prime}\left(q_{4}^{2}+q_{5}^{2}+q_{6}^{2}\right)\left(m_{1}^{2}+m_{2}^{2}+m_{3}^{2}\right) \\
& +\lambda_{\Gamma 4+\mathrm{m} \Gamma 4+}^{\prime \prime}\left(q_{4}^{2} m_{1}^{2}+q_{5}^{2} m_{2}^{2}+q_{6}^{2} m_{3}^{2}\right)+\frac{1}{4}\left(C_{11}^{\mathrm{o}}-C_{12}^{\mathrm{o}}\right)\left(e_{\mathrm{o}}^{2}+e_{\mathrm{t}}^{2}\right)+\frac{1}{6}\left(C_{11}^{\mathrm{o}}+2 C_{12}^{\mathrm{o}}\right) e_{\mathrm{a}}^{2}+\frac{1}{2} C_{44}^{\mathrm{o}}\left(e_{4}^{2}+e_{5}^{2}+e_{6}^{2}\right) \text {. }
\end{aligned}
$$

Here $a_{1}, a_{2}, b_{1}$, etc. are normal Landau coefficients, $T_{\mathrm{s} 1}$ and $T_{\mathrm{s} 2}$ are critical temperatures for the $X_{3}^{+}$and $\Gamma_{4}^{+}$tilting instabilities, $T_{\mathrm{c}}$ is the critical temperature for the magnetic instability, $\Theta_{\mathrm{s} 1}$, $\Theta_{\mathrm{s} 2}$, and $\Theta_{\mathrm{s} 3}$ are saturation temperatures, $\lambda_{1}, \lambda_{2}, \lambda_{3}, \lambda_{X 3+\Gamma 4+}$, $\lambda_{X 3+\mathrm{m} \Gamma 4+}^{\prime}$, etc. are coupling coefficients, and $\mathrm{C}_{11}^{\mathrm{o}}, \mathrm{C}_{12}^{\mathrm{o}}$, and $\mathrm{C}_{44}^{\mathrm{o}}$ are elastic constants of the reference cubic phase. Shear strains $e_{4}, e_{5}, e_{6}$ lie in the planes $Y Z, X Z$, and $X Y$, respectively, and the symmetry-adapted strains, $e_{\mathrm{a}}, e_{\mathrm{o}}$, and $e_{\mathrm{t}}$ are combinations of the linear strain components $e_{1}, e_{2}$, and $e_{3}$, as

$$
\begin{gathered}
e_{\mathrm{a}}=e_{1}+e_{2}+e_{3}, \\
e_{o}=\left(e_{1}-e_{2}\right), \\
e_{\mathrm{t}}=\frac{1}{\sqrt{3}}\left(2 e_{3}-e_{1}-e_{2}\right) .
\end{gathered}
$$

Direct coupling between order parameters from the different irreps is biquadratic, i.e., with the form $\lambda q^{2} m^{2}$ and $\lambda q^{2} q^{2}$, and could also occur indirectly via common strains. Table I gives solutions for different combinations of order parameter components with nonzero values, corresponding to different structural states which can arise due to tilting plus magnetic ordering in a parent structure which has $B$-site cation order and space group $F m \overline{3} m$.

The monoclinic structure of LNMO is due to the development of in-phase $\left(X_{3}^{+}\right)$and out-of-phase $\left(\Gamma_{4}^{+}\right)$octahedral tilt systems, while the $R \overline{3}$ structure arises as a consequence of out-of-phase $\left(\Gamma_{4}^{+}\right)$octahedral tilting alone [40,41]. Rogado et al. [5] have determined that the monoclinic structure is ferromagnetic with individual moments lined up parallel to the crystallographic $b$ axis, which corresponds to the [110] direction of the parent cubic structure. In the absence of any 
TABLE I. Subgroups of space group $F m \overline{3} m 1^{\prime}$ for irreps $X_{3}^{+}, \Gamma_{4}^{+}$and $m \Gamma_{4}^{+}$as the active representations.

\begin{tabular}{|c|c|c|c|c|c|}
\hline Subgroup & $X_{3}^{+}$ & $\Gamma_{4}^{+}$ & $m \Gamma_{4}^{+}$ & Lattice vectors & Origin \\
\hline$P 4 / m n c 1^{\prime}$ & $(a, 0,0)$ & $(0,0,0)$ & $(0,0,0)$ & $(-1 / 2,0,1 / 2),(1 / 2,0,1 / 2),(0,1,0)$ & $(0,0,0)$ \\
\hline$P 4_{2} / n n m 1^{\prime}$ & $(a, 0, a)$ & $(0,0,0)$ & $(0,0,0)$ & $(0,1,0),(0,0,1),(1,0,0)$ & $(0,0,0)$ \\
\hline Pnnn $1^{\prime}$ & $(a, b, c)$ & $(0,0,0)$ & $(0,0,0)$ & $(1,0,0),(0,1,0),(0,0,1)$ & $(0,0,0)$ \\
\hline$I 4 / m 1^{\prime}$ & $(0,0,0)$ & $(a, 0,0)$ & $(0,0,0)$ & $(0,1 / 2,1 / 2),(0,-1 / 2,1 / 2),(1,0,0)$ & $(0,0,0)$ \\
\hline$C 2 / m 1^{\prime}$ & $(0,0,0)$ & $(a, a, 0)$ & $(0,0,0)$ & $(-1 / 2,1 / 2,1),(-1 / 2,-1 / 2,0),(1 / 2,-1 / 2,0)$ & $(0,0,0)$ \\
\hline$P \overline{1} 1^{\prime}$ & $(0,0,0)$ & $(a, b, c)$ & $(0,0,0)$ & $(0,1 / 2,1 / 2),(1 / 2,0,1 / 2),(1 / 2,1 / 2,0)$ & $(0,0,0)$ \\
\hline$I 4 / \mathrm{mm}^{\prime} \mathrm{m}^{\prime}$ & $(0,0,0)$ & $(0,0,0)$ & $(a, 0,0)$ & $(0,1 / 2,-1 / 2),(0,1 / 2,1 / 2),(1,0,0)$ & $(0,0,0)$ \\
\hline$I m^{\prime} m^{\prime} m$ & $(0,0,0)$ & $(0,0,0)$ & $(a, a, 0)$ & $(0,0,-1),(-1 / 2,1 / 2,0),(1 / 2,1 / 2,0)$ & $(0,0,0)$ \\
\hline$R \overline{3} m^{\prime}$ & $(0,0,0)$ & $(0,0,0)$ & $(a, a, a)$ & $(1 / 2,0,-1 / 2),(0,-1 / 2,1 / 2),(-1,-1,-1)$ & $(0,0,0)$ \\
\hline$C 2^{\prime} / m^{\prime}$ & $(0,0,0)$ & $(0,0,0)$ & $(a, b, 0)$ & $(0,1,0),(0,0,-1),(-1 / 2,1 / 2,0)$ & $(0,0,0)$ \\
\hline$C 2^{\prime} / m^{\prime}$ & $(0,0,0)$ & $(0,0,0)$ & $(a, a, b)$ & $(1 / 2,1 / 2,1),(1 / 2,-1 / 2,0),(1 / 2,1 / 2,0)$ & $(0,0,0)$ \\
\hline$C 2^{\prime} / m^{\prime}$ & $(0,0,0)$ & $(a, 0,0)$ & $(0, b, c)$ & $(0,0,-1),(-1,0,0),(0,1 / 2,1 / 2)$ & $(0,0,0)$ \\
\hline$C 2^{\prime} / m^{\prime}$ & $(0,0,0)$ & $(a, a, 0)$ & $(b,-b,-c)$ & $(1 / 2,-1 / 2,-1),(-1 / 2,-1 / 2,0),(-1 / 2,1 / 2,0)$ & $(0,0,0)$ \\
\hline$P \overline{1}$ & $(0,0,0)$ & $(a, b, c)$ & $(d, e, f)$ & $(-1 / 2,-1 / 2,0),(-1 / 2,0,-1 / 2),(0,-1 / 2,-1 / 2)$ & $(0,0,0)$ \\
\hline$R \overline{3}$ & $(a,-a, a)$ & $(b, b, b)$ & $(0,0,0)$ & $(0,1,-1),(-1,0,1),(1,1,1)$ & $(0,1 / 2,1 / 2)$ \\
\hline$C 2 / c$ & $(a, 0,0)$ & $(b, 0,0)$ & $(0,0,0)$ & $(0,0,1),(1,0,0),(0,1,0)$ & $(1 / 4,0,1 / 4)$ \\
\hline$P 4 / m$ & $(a, 0,0)$ & $(0, b, 0)$ & $(0,0,0)$ & $(1 / 2,0,1 / 2),(1 / 2,0,-1 / 2),(0,1,0)$ & $(0,0,0)$ \\
\hline$P 4_{2} / n$ & $(a, 0, a)$ & $(b, 0,0)$ & $(0,0,0)$ & $(0,1,0),(0,0,1),(1,0,0)$ & $(-1 / 2,0,-1 / 2)$ \\
\hline$P 2 / c$ & $(a, b, c)$ & $(d, 0,0)$ & $(0,0,0)$ & $(0,0,1),(-1,0,0),(0,-1,-1)$ & $(0,0,0)$ \\
\hline$P 2_{1} / c$ & $(a, 0,0)$ & $(b, 0, b)$ & $(0,0,0)$ & $(-1 / 2,0,1 / 2),(-1 / 2,0,-1 / 2),(1 / 2,-1,-1 / 2)$ & $(0,0,0)$ \\
\hline$C 2 / m$ & $(a, 0, a)$ & $(0, b, b)$ & $(0,0,0)$ & $(0,1,-1),(0,1,1),(1,0,0)$ & $(0,0,0)$ \\
\hline$P \overline{1}$ & $(a, 0,0)$ & $(b, c, d)$ & $(0,0,0)$ & $(1 / 2,0,1 / 2),(1 / 2,0,-1 / 2),(0,1,0)$ & $(0,0,0)$ \\
\hline$R \overline{3}$ & $(a-a, a)$ & $(0,0,0)$ & $(b, b, b)$ & $(1,-1,0),(-1,0,1),(-1,-1,-1)$ & $(-1 / 2,0,0)$ \\
\hline$C 2^{\prime} / c^{\prime}$ & $(a, 0,0)$ & $(0,0,0)$ & $(b, c, 0)$ & $(-1,0,0),(0,0,1),(0,1,0)$ & $(-1 / 4,0,1 / 4)$ \\
\hline$P 2^{\prime} / m^{\prime}$ & $(a, 0,0)$ & $(0,0,0)$ & $(b, 0, c)$ & $(1 / 2,0,-1 / 2),(0,1,0),(1 / 2,0,1 / 2)$ & $(0,0,0)$ \\
\hline$P 2^{\prime} / c^{\prime}$ & $(a, b, c)$ & $(0,0,0)$ & $(d, \mathrm{e}, 0)$ & $(-1,0,0),(0,0,-1),(1,-1,0)$ & $(0,0,0)$ \\
\hline$P 2_{1}^{\prime} / c^{\prime}$ & $(a, 0,0)$ & $(0,0,0)$ & $(b, c, b)$ & $(1 / 2,0,1 / 2),(-1 / 2,0,1 / 2),(-1 / 2,-1,-1 / 2)$ & $(0,0,0)$ \\
\hline$C 2^{\prime} / m^{\prime}$ & $(a, 0, a)$ & $(0,0,0)$ & $(b, c, c)$ & $(0,1,1),(0,1,-1),(-1,0,0)$ & $(0,1 / 2,0)$ \\
\hline$P \overline{1}$ & $(a, 0,0)$ & $(0,0,0)$ & $(b, c, d)$ & $(1 / 2,0,1 / 2),(1 / 2,0,-1 / 2),(0,-1,0)$ & $(0,0,0)$ \\
\hline$P \overline{1}$ & $(a, b, c)$ & $(0,0,0)$ & $(d, e, f)$ & $(0,1,0),(0,0,1),(1,0,0)$ & $(0,0,0)$ \\
\hline$C 2 / c$ & $(a, 0,0)$ & $(b, 0,0)$ & $(c, 0,0)$ & $(0,0,1),(1,0,0),(0,1,0)$ & $(1 / 4,0,1 / 4)$ \\
\hline$P 4 / m$ & $(a, 0,0)$ & $(0, b, 0)$ & $(0, c, 0)$ & $(1 / 2,0,1 / 2),(1 / 2,0,-1 / 2),(0,1,0)$ & $(0,0,0)$ \\
\hline$P 4_{2} / n$ & $(a, 0, a)$ & $(b, 0,0)$ & $(c, 0,0)$ & $(0,1,0),(0,0,1),(1,0,0)$ & $(-1 / 2,0,-1 / 2)$ \\
\hline$P 2 / c$ & $(a, b, c)$ & $(d, 0,0)$ & $(e, 0,0)$ & $(0,0,1),(-1,0,0),(0,-1,-1)$ & $(0,0,0)$ \\
\hline$P 2_{1} / c$ & $(a, 0,0)$ & $(b, 0, b)$ & $(c, 0, c)$ & $(-1 / 2,0,1 / 2),(-1 / 2,0,-1 / 2),(1 / 2,-1,-1 / 2)$ & $(0,0,0)$ \\
\hline$C 2 / m$ & $(a, 0, a)$ & $(0, b, b)$ & $(0, c, c)$ & $(0,1,-1),(0,1,1),(1,0,0)$ & $(0,0,0)$ \\
\hline$R \overline{3}$ & $(a,-a, a)$ & $(b, b, b)$ & $(c, c, c)$ & $(1,-1,0),(-1,0,1),(-1,-1,-1)$ & $(-1 / 2,0,0)$ \\
\hline$C 2^{\prime} / c^{\prime}$ & $(a, 0,0)$ & $(b, 0,0)$ & $(0, c, d)$ & $(0,0,1),(1,0,0),(0,1,0)$ & $(1 / 4,0,1 / 4)$ \\
\hline$P 2^{\prime} / m^{\prime}$ & $(a, 0,0)$ & $(0, b, 0)$ & $(c, 0, d)$ & $(1 / 2,0,-1 / 2),(0,1,0),(1 / 2,0,1 / 2)$ & $(0,0,0)$ \\
\hline$P 2^{\prime} / c^{\prime}$ & $(a, b, c)$ & $(d, 0,0)$ & $(0, e, f)$ & $(0,0,1),(-1,0,0),(0,-1,-1)$ & $(0,0,0)$ \\
\hline$P 2_{1}^{\prime} / c^{\prime}$ & $(a, 0,0)$ & $(b, 0, b)$ & $(c,-d,-c)$ & $(-1 / 2,0,1 / 2),(-1 / 2,0,-1 / 2),(1 / 2,-1,-1 / 2)$ & $(0,0,0)$ \\
\hline$C 2^{\prime} / m^{\prime}$ & $(a, 0, a)$ & $(0, b, b)$ & $(-c, d,-d)$ & $(0,1,-1),(0,1,1),(1,0,0)$ & $(0,0,0)$ \\
\hline$P \overline{1}$ & $(a, 0,0)$ & $(b, c, d)$ & $(e, f, g)$ & $(1 / 2,0,1 / 2),(1 / 2,0,-1 / 2),(0,1,0)$ & $(0,0,0)$ \\
\hline$P \overline{1}$ & $(a, b, c)$ & $(d, e, f)$ & $(g, h, i)$ & $(0,1,0),(0,0,1),(1,0,0)$ & $(0,0,0)$ \\
\hline
\end{tabular}


tilting, the space group for the analogous FM structure would be $I 4 / m m^{\prime} m^{\prime}, I m^{\prime} m^{\prime} m$ or $R \overline{3} m$ (Table I). The FM moments of the rhombohedral structure will be aligned parallel to [111] of the parent cubic structure and the magnetic space group will be $R \overline{3}$.
To get the monoclinic structure in the $P 2_{1} / n$ setting, rather than in the standard $P 2_{1} / c$ setting given in Table $\mathrm{I}$, it is convenient to choose direction $P 1(3)$ of $X_{3}^{+}$and direction $P 2(1)$ of $\Gamma_{4}^{+}$which leads to $q_{1}=q_{2}=0, q_{3} \neq 0, q_{4}=q_{5} \neq 0$, $q_{6}=0, m_{1}=m_{2} \neq 0, m_{3}=0$. Equation (A1) then reduces to

$$
\begin{aligned}
G= & \frac{1}{2} a_{1} \Theta_{\mathrm{s} 1}\left(\operatorname{coth}\left(\frac{\Theta_{\mathrm{s} 1}}{T}\right)-\operatorname{coth}\left(\frac{\Theta_{\mathrm{s} 1}}{T_{\mathrm{s} 1}}\right)\right) q_{3}^{2}+\frac{1}{4}\left(b_{1}+b_{1}^{\prime}\right) q_{3}^{4}+a_{2} \Theta_{\mathrm{s} 2}\left(\operatorname{coth}\left(\frac{\Theta_{\mathrm{s} 2}}{T}\right)-\operatorname{coth}\left(\frac{\Theta_{\mathrm{s} 2}}{T_{\mathrm{s} 2}}\right)\right) q_{4}^{2} \\
& +\frac{1}{2}\left(2 b_{2}+b_{2}^{\prime}\right) q_{4}^{4}+a_{3} \Theta_{\mathrm{s} 3}\left(\operatorname{coth}\left(\frac{\Theta_{\mathrm{s} 3}}{T}\right)-\operatorname{coth}\left(\frac{\Theta_{\mathrm{s} 3}}{T_{\mathrm{c}}}\right)\right) m_{1}^{2}+\frac{1}{2}\left(2 b_{3}+b_{3}^{\prime}\right) m_{1}^{4}+e_{\mathrm{a}}\left(\lambda_{1} q_{3}^{2}+2 \lambda_{2} q_{4}^{2}+2 \lambda_{3} m_{1}^{2}\right) \\
& +2 e_{\mathrm{t}}\left(\lambda_{4} q_{3}^{2}-\lambda_{5} q_{4}^{2}-\lambda_{6} m_{1}^{2}\right)+\lambda_{7}\left(e_{4}^{2}+e_{5}^{2}+e_{6}^{2}\right) q_{3}^{2}+\lambda_{8}\left(e_{4}^{2}+e_{5}^{2}\right) q_{3}^{2}+e_{6}\left(\lambda_{9} q_{4}^{2}+\lambda_{11} m_{1}^{2}\right)+\lambda_{10}\left(e_{5}-e_{4}\right) q_{3}^{2} q_{4} \\
& +2\left(\lambda_{X 3+\Gamma 4+}+\lambda_{X 3+\Gamma 4+}^{\prime}\right) q_{3}^{2} q_{4}^{2}+2\left(\lambda_{X 3+\mathrm{m} \Gamma 4+}+\lambda_{X 3+m \Gamma 4+}^{\prime}\right) q_{3}^{2} m_{1}^{2}+2\left(2 \lambda_{\Gamma 4+\mathrm{m} \Gamma 4+}+2 \lambda_{\Gamma 4+\mathrm{m} \Gamma 4+}^{\prime}+\lambda_{\Gamma 4+\mathrm{m} \Gamma 4+}^{\prime \prime}\right) q_{4}^{2} m_{1}^{2} \\
& +\frac{1}{4}\left(C_{11}^{\mathrm{o}}-C_{12}^{\mathrm{o}}\right)\left(e_{\mathrm{o}}^{2}+e_{\mathrm{t}}^{2}\right)+\frac{1}{6}\left(C_{11}^{\mathrm{o}}+2 C_{12}^{\mathrm{o}}\right) e_{\mathrm{a}}^{2}+\frac{1}{2} C_{44}^{\mathrm{o}}\left(e_{4}^{2}+e_{5}^{2}+e_{6}^{2}\right) .
\end{aligned}
$$

The equilibrium condition $\partial \mathrm{G} / \partial \mathrm{e}=0$ gives relationships between strains and order parameter components as

$$
\begin{gathered}
e_{\mathrm{a}}=-\frac{\lambda_{1} q_{3}^{2}+2 \lambda_{2} q_{4}^{2}+2 \lambda_{3} m_{1}^{2}}{\frac{1}{3}\left(C_{11}^{\mathrm{o}}+2 C_{12}^{\mathrm{o}}\right)}, \\
e_{\mathrm{t}}=-\frac{2\left(\lambda_{4} q_{3}^{2}-\lambda_{5} q_{4}^{2}-\lambda_{6} m_{1}^{2}\right)}{\frac{1}{2}\left(C_{11}^{\mathrm{o}}-C_{12}^{\mathrm{o}}\right)}, \\
e_{o}=0, \\
e_{5}=-e_{4}=-\frac{\lambda_{10} q_{3}^{2} q_{4}}{2\left(\lambda_{7}+\lambda_{8}\right) q_{3}^{2}+\mathrm{C}_{44}^{\mathrm{o}}} \approx-\frac{\lambda_{10} q_{3}^{2} q_{4}}{\mathrm{C}_{44}^{\mathrm{o}}} \text { for } \mathrm{C}_{44}^{\mathrm{o}} \gg 2\left(\lambda_{7}+\lambda_{8}\right) q_{3}^{2}, \\
e_{6}=-\frac{\lambda_{9} q_{4}^{2}+\lambda_{11} m_{1}^{2}}{2 \lambda_{7} q_{3}^{2}+\mathrm{C}_{44}^{\mathrm{o}}} \approx-\frac{\lambda_{9} q_{4}^{2}+\lambda_{11} m_{1}^{2}}{\mathrm{C}_{44}^{\mathrm{o}}} \text { for } \mathrm{C}_{44}^{\mathrm{o}} \gg 2 \lambda_{7} q_{3}^{2} .
\end{gathered}
$$

The $R \overline{3}$ structure has $q_{1}=q_{2}=q_{3}=0, q_{4}=q_{5}=q_{6} \neq 0, m_{1}=m_{2}=m_{3} \neq 0$, and Eq. (A1) reduces to

$$
\begin{aligned}
G= & \frac{3}{2} a_{2} \Theta_{\mathrm{s} 2}\left(\operatorname{coth}\left(\frac{\Theta_{\mathrm{s} 2}}{T}\right)-\operatorname{coth}\left(\frac{\Theta_{\mathrm{s} 2}}{T_{\mathrm{s} 2}}\right)\right) q_{4}^{2}+\frac{1}{4}\left(9 b_{2}+3 b_{2}^{\prime}\right) q_{1}^{4}+\frac{3}{2} a_{3} \Theta_{\mathrm{s} 3}\left(\operatorname{coth}\left(\frac{\Theta_{\mathrm{s} 3}}{T}\right)-\operatorname{coth}\left(\frac{\Theta_{\mathrm{s} 3}}{T_{\mathrm{c}}}\right)\right) m_{1}^{2} \\
& +\frac{1}{4}\left(9 b_{2}+3 b_{2}^{\prime}\right) m_{1}^{4}+3 e_{\mathrm{a}}\left(\lambda_{2} q_{4}^{2}+\lambda_{3} m_{1}^{2}\right)+\left(e_{4}+e_{5}+e_{6}\right)\left(\lambda_{9} q_{4}^{2}+\lambda_{11} m_{1}^{2}\right)+3 q_{4}^{2} m_{1}^{2}\left(3 \lambda_{\Gamma 4+\mathrm{m} \Gamma 4+}+3 \lambda_{\Gamma 4+\mathrm{m} \Gamma 4+}^{\prime}\right. \\
& \left.+\lambda_{\Gamma 4+\mathrm{m} \Gamma 4+}^{\prime \prime}\right)+\frac{1}{4}\left(\mathrm{C}_{11}^{\mathrm{o}}-\mathrm{C}_{12}^{\mathrm{o}}\right)\left(e_{2}^{\mathrm{o}}+e_{\mathrm{t}}^{2}\right)+\frac{1}{6}\left(\mathrm{C}_{11}^{\mathrm{o}}+2 \mathrm{C}_{12}^{\mathrm{o}}\right) e_{\mathrm{a}}^{2}+\frac{1}{2} \mathrm{C}_{44}^{\mathrm{o}}\left(e_{4}^{2}+e_{5}^{2}+e_{6}^{2}\right) .
\end{aligned}
$$

The equilibrium condition $\partial \mathrm{G} / \partial \mathrm{e}=0$ then gives relationships between strains and order parameter components as

$$
\begin{gathered}
e_{\mathrm{a}}=-\frac{3\left(\lambda_{2} q_{4}^{2}+\lambda_{3} m_{1}^{2}\right)}{\frac{1}{3}\left(\mathrm{C}_{11}^{\mathrm{o}}+2 \mathrm{C}_{12}^{\mathrm{o}}\right)}, \\
e_{\mathrm{t}}=e_{\mathrm{o}}=0, \\
e_{4}=e_{5}=e_{6}=-\frac{\lambda_{9} q_{4}^{2}+\lambda_{11} m_{1}^{2}}{\mathrm{C}_{44}^{\mathrm{o}}} .
\end{gathered}
$$

These strain/order parameter relationships provide a straightforward means of analyzing the evolution of the different order parameters. To this end, lattice parameter data from neutron and x-ray-diffraction measurements at temperatures in the range 80-1073 K [37] are reproduced in Fig. 9(a) in terms of pseudocubic parameters with respect to the parent $F m \overline{3} m$ structure. This sample had been annealed in air at $1523 \mathrm{~K}$ for $24 \mathrm{~h}$ and then at $1123 \mathrm{~K}$ for $8 \mathrm{~h}$. The rhombohedral phase had $Q_{\text {od }} \approx 0.48$ and the monoclinic phase had $Q_{\text {od }} \approx 0.3$, based on refined $B$-site occupancies. In order to determine the spontaneous strain variations, it is necessary to define a reference lattice parameter, $a_{0}$, for the high-symmetry structure, extrapolated through the stability fields of the lowsymmetry structures (e.g., see Carpenter et al. [87]). Only data for the rhombohedral and monoclinic structures are available, so this approach is not possible in the present case. Instead, shear strains can be determined without significant loss of precision by using the approximation $a_{\mathrm{o}}=V^{1 / 3}$, where $V$ is the 


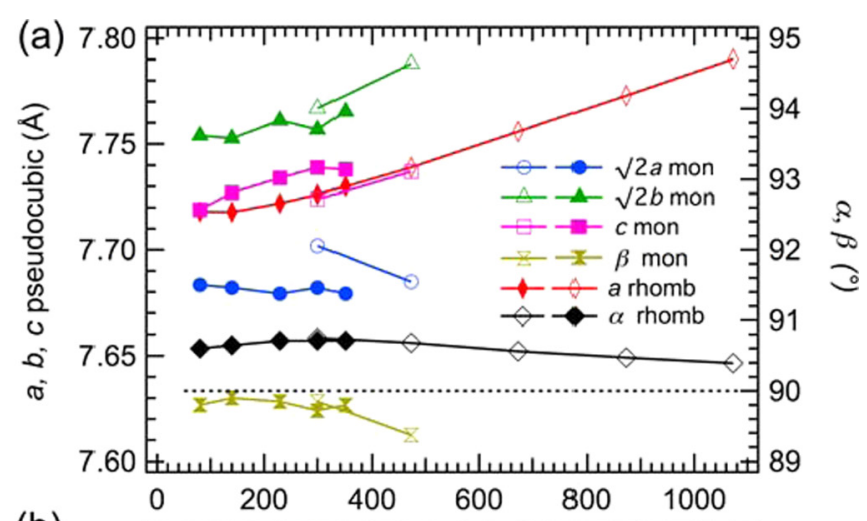

pseudocubic unit-cell volume of the low-symmetry structures. The chosen setting and orientation of the $P 2_{1} / n$ cell has lattice vectors $(-1 / 2,1 / 2,0),(1 / 2,1 / 2,0),(0,0,-1)$ with respect to the parent $F m \overline{3} m$ structure. At room temperature, the lattice parameters are $a=5.43 \AA, b=5.49 \AA, c=7.74 \AA$, $\beta=89.7^{\circ}$ [37]. Non-zero strains for the monoclinic structure are given by

$$
\begin{gathered}
e_{1}+e_{2}=\frac{\sqrt{2} a-a_{\mathrm{o}}}{a_{\mathrm{o}}}+\frac{\sqrt{2} b-a_{\mathrm{o}}}{a_{\mathrm{o}}}, \\
e_{3}=\frac{c-a_{\mathrm{o}}}{a_{\mathrm{o}}} \\
\left(e_{5}-e_{4}\right) \approx \cos \beta \\
e_{6}=\frac{\sqrt{2} a-a_{\mathrm{o}}}{a_{\mathrm{o}}}-\frac{\sqrt{2} b-a_{\mathrm{o}}}{a_{\mathrm{o}}} .
\end{gathered}
$$

In the case of the rhombohedral structure, the only non-zero shear strain is $e_{4}\left(=e_{5}=e_{6}\right)$, which is given by

$$
e_{4} \approx \cos \alpha \text {. }
$$

Variations of individual shear strains with temperature are shown in Fig. 9(b). $e_{4}$ scales with $q_{4}^{2}$ above the magnetic ordering transition and has a linear temperature dependence consistent with a second-order $F m \overline{3} m-R \overline{3}$ transition and an extrapolated transition temperature of $\sim 1880 \mathrm{~K}$, as previously reported by Yang et al. [39]. Shear strains $e_{4}$ of the $R \overline{3}$ structure and $e_{6}$ of the $P 2_{1} / n$ structure both depend on $q_{4}^{2}$ and $m_{1}^{2}$ [Eqs. (A10), (A14)] and the reduction of their values below $\sim 300 \mathrm{~K}$ indicates that there is unfavorable coupling between the tilt and magnetic order parameters. Values of $e_{\mathrm{t}}$ are small for the $P 2_{1} / n$ structure and reduce with falling temperature because couplings with the two tilt systems have opposite sign [Eq. (A7)]. At the resolution of the data, they do not provide any further indication of the strength of coupling with the magnetic order parameter. The additional nonzero strain of the $P 2_{1} / n$ structure, $e_{5}\left(=-e_{4}\right)$, arises from coupling terms with the symmetry combination $X_{3}^{+} \oplus \Gamma_{4}^{+} \oplus \Gamma_{5}^{+}$which is allowed in the Landau expansion. This scales with $\lambda_{10} q_{3}^{2} q_{4}$ [Eq. (A9)], such that reductions in tilt angles due to unfavorable coupling with the magnetic order parameter should appear as a decrease in the $\beta$ angle below the magnetic ordering temperature-as is the case on the basis of the limited data in Figs. 9(a) and 9 (b).

Unit-cell volume variations are shown in Fig. 9(c) and suggest that there might be a small reduction associated with the first-order $R \overline{3} \rightarrow P 2_{1} / n$ transition. It is possible that there is a change in volume also with magnetic ordering but the nonlinearity below $\sim 300 \mathrm{~K}$ could be due simply to the normal saturation of thermal expansion as temperature tends to $0 \mathrm{~K}$.

[1] S. Vasala and M. Karppinen, Prog. Solid State Chem. 43, 1 (2015).

[2] D. Serrate, J. M. De Teresa, and M. R. Ibarra, J. Phys.: Condens. Matter 19, 023201 (2007).

[3] K. I. Kobayashi, T. Kimura, H. Sawada, K. Terakura, and Y. Tokura, Nature (London) 395, 677 (1998).

[4] X. W. Jin, L. Lu, S. B. Mi, M. Liu, and C. L. Jia, Appl. Phys. Lett. 109, 031904 (2016). 
[5] N. S. Rogado, J. Li, A. W. Sleight, and M. A. Subramanian, Adv. Mater. 17, 2225 (2005).

[6] D. Choudhury, P. Mandal, R. Mathieu, A. Hazarika, S. Rajan, A. Sundaresan, U. V. Waghmare, R. Knut, O. Karis, P. Nordblad, and D. D. Sarma, Phys. Rev. Lett. 108, 127201 (2012)

[7] K. Devi Chandrasekhar, A. K. Das, C. Mitra, and A. Venimadhav, J. Phys.: Condens. Matter 24, 495901 (2012).

[8] M. Sariful Sheikh, D. Ghosh, A. Dutta, S. Bhattacharyya, and T. P. Sinha, Mater. Sci. Eng.: B 226, 10 (2017).

[9] C. Lan, S. Zhao, T. Xu, J. Ma, S. Hayase, and T. Ma, J. Alloys Compd. 655, 208 (2016).

[10] R. I. Dass, J.-Q. Yan, and J. B. Goodenough, Phys. Rev. B 68, 064415 (2003).

[11] M. Iliev, H. Guo, and A. Gupta, Appl. Phys. Lett. 90, 151914 (2007)

[12] C. Bull, D. Gleeson, and K. Knight, J. Phys.: Condens. Matter 15, 4927 (2003).

[13] J. B. Goodenough, Phys. Rev. 100, 564 (1955).

[14] R. Takahashi, I. Ohkubo, K. Yamauchi, M. Kitamura, Y. Sakurai, M. Oshima, T. Oguchi, Y. Cho, and M. Lippmaa, Phys. Rev. B 91, 134107 (2015).

[15] D. Yang, R. J. Harrison, J. A. Schiemer, G. I. Lampronti, X. Liu, F. Zhang, H. Ding, Y. Liu, and M. A. Carpenter, Phys. Rev. B 93, 024101 (2016).

[16] H. Q. Yin, J. S. Zhou, R. Dass, J. P. Zhou, J. T. Mcdevitt, and J. B. Goodenough, J. Appl. Phys. 87, 6761 (2000).

[17] C. Du, R. Adur, H. Wang, A. J. Hauser, F. Yang, and P. C. Hammel, Phys. Rev. Lett. 110, 147204 (2013).

[18] H. Deniz, D. Preziosi, M. Alexe, D. Hesse, C. Eisenschmidt, G. Schmidt, and L. Pintilie, J. Mater. Sci. 50, 3131 (2015).

[19] T. Fix, D. Stoeffler, S. Colis, C. Ulhaq, G. Versini, J. P. Vola, F. Huber, and A. Dinia, J. Appl. Phys. 98, 023712 (2005).

[20] H. Deniz, D. Preziosi, M. Alexe, and D. Hesse, J. Appl. Phys. 121, 023906 (2017).

[21] M. Hashisaka, D. Kan, A. Masuno, M. Takano, Y. Shimakawa, T. Terashima, and K. Mibu, Appl. Phys. Lett. 89, 032504 (2006).

[22] M. Kitamura, I. Ohkubo, M. Kubota, Y. Matsumoto, H. Koinuma, and M. Oshima, Appl. Phys. Lett. 94, 132506 (2009).

[23] M. Kitamura, I. Ohkubo, M. Matsunami, K. Horiba, H. Kumigashira, Y. Matsumoto, H. Koinuma, and M. Oshima, Appl. Phys. Lett. 94, 262503 (2009).

[24] M. P. Singh, C. Grygiel, W. C. Sheets, P. Boullay, M. Hervieu, W. Prellier, B. Mercey, C. Simon, and B. Raveau, Appl. Phys. Lett. 91, 012503 (2007).

[25] M. P. Singh, K. D. Truong, S. Jandl, and P. Fournier, Phys. Rev. B 79, 224421 (2009).

[26] K. D. Truong, M. P. Singh, S. Jandl, and P. Fournier, Phys. Rev. B 80, 134424 (2009).

[27] H. J. Zhao, W. Ren, Y. Yang, J. Iniguez, X. M. Chen, and L. Bellaiche, Nat Commun 5, 4021 (2014).

[28] Y. Sakurai, I. Ohkubo, Y. Matsumoto, H. Koinuma, and M. Oshima, J. Appl. Phys. 110, 063913 (2011).

[29] S. Kazan, F. A. Mikailzade, M. Özdemir, B. Aktaş, B. Rameev, A. Intepe, and A. Gupta, Appl. Phys. Lett. 97, 072511 (2010).

[30] S. Q. Wu, S. Cheng, L. Lu, M. Liu, X. W. Jin, S. D. Cheng, and S. B. Mi, Sci. Rep. 8, 2516 (2018).

[31] H. Das, U. V. Waghmare, T. Saha-Dasgupta, and D. D. Sarma, Phys. Rev. Lett. 100, 186402 (2008).
[32] F. Wei, Z. Deng, S. Sun, F. Xie, G. Kieslich, D. M. Evans, M. A. Carpenter, P. D. Bristowe, and A. K. Cheetham, Mater. Horiz. 3, 328 (2016).

[33] J. Blasco, J. García, M. C. Sánchez, J. Campo, G. Subías, and J. Pérez-Cacho, Eur. Phys. J. B 30, 469 (2002).

[34] D. A. B. Barbosa, M. W. Lufaso, H. Reichlova, X. Marti, M. V. S. Rezende, A. P. Maciel, and C. W. A. Paschoal, J. Alloys Compd. 663, 899 (2016).

[35] V. L. Joseph Joly, P. A. Joy, S. K. Date, and C. S. Gopinath, Phys. Rev. B 65, 184416 (2002).

[36] M. C. Sánchez, J. García, J. Blasco, G. Subías, and J. PerezCacho, Phys. Rev. B 65, 144409 (2002).

[37] F. N. Sayed, S. N. Achary, O. D. Jayakumar, S. K. Deshpande, P. S. R. Krishna, S. Chatterjee, P. Ayyub, and A. K. Tyagi, J. Mater. Res. 26, 567 (2011).

[38] P. Kumar, S. Ghara, B. Rajeswaran, D. Muthu, A. Sundaresan, and A. Sood, Solid State Commun. 184, 47 (2014).

[39] D. Yang, W. Wang, T. Yang, G. I. Lampronti, H. Ye, L. Wu, Q. Yu, and S. Lu, APL Materials 6, 066102 (2018).

[40] C. J. Howard, B. J. Kennedy, and P. M. Woodward, Acta Crystallogr., Sect. B 59, 463 (2003).

[41] M. Gateshki, J. Igartua, and E. Hernandez-Bocanegra, J. Phys. Condens. Matter 15, 6199 (2003).

[42] J. Blasco, M. C. Sánchez, J. Pérez-Cacho, J. García, G. Subías, and J. Campo, J. Phys. Chem. Solids 63, 781 (2002).

[43] H. Z. Guo, J. Burgess, E. Ada, S. Street, A. Gupta, M. N. Iliev, A. J. Kellock, C. Magen, M. Varela, and S. J. Pennycook, Phys. Rev. B 77, 174423 (2008).

[44] Y. Q. Lin, X. M. Chen, and X. Q. Liu, Solid State Commun. 149, 784 (2009).

[45] Z. Zhang, H. Jian, X. Tang, J. Yang, X. Zhu, and Y. Sun, Dalton Trans. 41, 11836 (2012).

[46] H. Guo, A. Gupta, M. Varela, S. Pennycook, and J. Zhang, Phys. Rev. B 79, 172402 (2009).

[47] S. Zhao, L. Shi, S. Zhou, J. Zhao, H. Yang, and Y. Guo, J. Appl. Phys. 106, 123901 (2009).

[48] M. N. Iliev, M. M. Gospodinov, M. P. Singh, J. Meen, K. D. Truong, P. Fournier, and S. Jandl, J. Appl. Phys. 106, 023515 (2009).

[49] M. G. Masud, A. Ghosh, J. Sannigrahi, and B. K. Chaudhuri, J Phys.: Condens. Matter 24, 295902 (2012).

[50] M. P. Reddy, R. A. Shakoor, and A. M. A. Mohamed, Mater. Chem. Phys. 177, 346 (2016).

[51] Y. Guo, L. Shi, S. Zhou, J. Zhao, and W. Liu, Appl. Phys. Lett. 102, 222401 (2013).

[52] J. Maynard, Phys. Today 49(1), 26 (1996).

[53] A. Migliori, J. L. Sarrao, W. M. Visscher, T. M. Bell, M. Lei, Z. Fisk, and R. G. Leisure, Physica B 183, 1 (1993).

[54] R. G. Leisure and F. A. Willis, J. Phys.: Condens. Matter 9, 6001 (1997).

[55] B. J. Zadler, J. H. L. Le Rousseau, J. A. Scales, and M. L. Smith, Geophys. J. Int. 156, 154 (2004).

[56] R. S. Lakes, Rev. Sci. Instrum. 75, 797 (2004).

[57] A. Migliori and J. D. Maynard, Rev. Sci. Instrum. 76, 121301 (2005)

[58] R. E. A. McKnight, M. A. Carpenter, T. W. Darling, A. Buckley, and P. A. Taylor, Am. Mineral. 92, 1665 (2007).

[59] R. E. McKnight, T. Moxon, A. Buckley, P. Taylor, T. Darling, and M. Carpenter, J. Phys.: Condens. Matter 20, 075229 (2008). 
[60] C. L. Bull and P. F. McMillan, J. Solid State Chem. 177, 2323 (2004).

[61] D. A. B. Barbosa and C. W. A. Paschoal, Spectrochim. Acta Part A 185, 125 (2017).

[62] M. N. Iliev, M. V. Abrashev, A. P. Litvinchuk, V. G. Hadjiev, H. Guo, and A. Gupta, Phys. Rev. B 75, 104118 (2007).

[63] E. K. H. Salje, Phase Transitions 37, 83 (1992).

[64] E. K. H. Salje and U. Bismayer, Phase Transitions 63, 1 (1997).

[65] E. K. H. Salje, in Feldspars and their Reactions, NATO ASI Series, edited by I. Parsons (Springer, Netherlands, Dordrecht, 1994), p. 103.

[66] E. Granado, A. García, J. A. Sanjurjo, C. Rettori, I. Torriani, F. Prado, R. D. Sánchez, A. Caneiro, and S. B. Oseroff, Phys. Rev. B 60, 11879 (1999).

[67] J. Laverdière, S. Jandl, A. A. Mukhin, V. Y. Ivanov, V. G. Ivanov, and M. N. Iliev, Phys. Rev. B 73, 214301 (2006).

[68] D. Yang, T. Chatterji, J. A. Schiemer, and M. A. Carpenter, Phys. Rev. B 93, 144109 (2016).

[69] C. Salazar Mejía, N. O. Born, J. A. Schiemer, C. Felser, M. A. Carpenter, and M. Nicklas, Phys. Rev. B 97, 094410 (2018).

[70] L. Zhang, X. Ren, and M. A. Carpenter, Phys. Rev. B 95, 054116 (2017).

[71] L. Zhang, X. Lou, D. Wang, Y. Zhou, Y. Yang, M. Kuball, M. A. Carpenter, and X. Ren, Phys. Rev. Appl. 8, 054018 (2017).

[72] M. A. Carpenter, J. A. Schiemer, I. Lascu, R. J. Harrison, A. Kumar, R. S. Katiyar, N. Ortega, D. A. Sanchez, C. S. Mejia, W. Schnelle, M. Echizen, H. Shinohara, A. J. F. Heap, R. Nagaratnam, S. E. Dutton, and J. F. Scott, J. Phys.: Condens. Matter 27, 285901 (2015).
[73] M. Carpenter, J. Phys.: Condens. Matter 27, 263201 (2015).

[74] E. A. M. Ruth, C. J. Howard, and M. A. Carpenter, J. Phys.: Condens. Matter 21, 015901 (2009).

[75] Z. Zhang, J. Koppensteiner, W. Schranz, J. B. Betts, A. Migliori, and M. A. Carpenter, Phys. Rev. B 82, 014113 (2010).

[76] N. J. Perks, Z. Zhang, R. J. Harrison, and M. A. Carpenter, J. Phys.: Condens. Matter 26, 505402 (2014).

[77] M. Carpenter, A. Buckley, P. Taylor, and T. Darling, J. Phys.: Condens. Matter 22, 035405 (2010).

[78] R. J. Harrison and S. A. T. Redfern, Phys. Earth Planet. Inter. 134, 253 (2002).

[79] R. I. Thomson, T. Chatterji, and M. A. Carpenter, J. Phys.: Condens. Matter 26, 146001 (2014).

[80] D. Yang, T. Yang, P. Mukherjee, S. E. Dutton, D. Huo, and M. A. Carpenter, Phys. Rev. B 99, 094314 (2019).

[81] M. A. Carpenter and E. K. H. Salje, Eur. J. Mineral. 10, 693 (1998).

[82] J. Chrosch and E. K. H. Salje, J. Appl. Phys. 85, 722 (1999).

[83] S. A. Hayward, J. Chrosch, E. K. H. Salje, and M. A. Carpenter, Eur. J. Mineral. 8, 1301 (1997).

[84] M. A. Carpenter and Z. Zhang, Geophys. J. Int. 186, 279 (2011).

[85] E. K. H. Salje, X. Ding, Z. Zhao, T. Lookman, and A. Saxena, Phys. Rev. B 83, 104109 (2011).

[86] Z. Zhang, J. Koppensteiner, W. Schranz, D. Prabhakaran, and M. A. Carpenter, J. Phys.: Condens. Matter 23, 145401 (2011).

[87] D. Pesquera, M. A. Carpenter, and E. K. H. Salje, Phys. Rev. Lett. 121, 235701 (2018). 